\title{
Syntax friendly and syntax amendment approaches to error resiliency in the MPEG-4 Simple Scalable Profile
}

\author{
Tamer Shanableh ${ }^{1}$ \\ American University of Sharjah \\ Po. Box 26666, UAE \\ tshanableh@ausharjah.edu
}

\author{
Gus Reid \\ Motorola Labs, Jays Close, \\ Basingstoke, Hampshire RG22 4PD, UK \\ Gus.Reid@motorola.com
}

https://doi.org/10.1016/j.image.2005.03.009

\section{Abstract}

This work discusses two approaches to incorporating error resiliency in the Simple Scalable Profile SSP of the ISO/IEC MPEG-4 14496-2 visual standard. As such scalable MPEG-4 is made suitable for deployment in a mobile communication environment. When it was defined in 2000, the syntax of the Simple Scalable Profile (SSP) prohibited the use of the error resiliency tools which were available in the base layer of the MPEG-4 codec. GPRS simulations are employed to illustrate the need for error resiliency in the SSP profile. Two solutions are then proposed based on whether or not bitstream syntax modifications are employed. The first solution is syntax friendly and emulates the use of the Video Packet resiliency tool whilst remaining compliant with standardized decoders. The limitations and constraints of such an approach are then elaborated upon. It is shown that syntax modifications are inevitable. Thus the second solution proposes the syntax modifications to incorporate the error resiliency tools of the base layer into the enhancement layers of the SSP profile. The new syntax is verified through subjective testing using Multi Media Double Stimulus Continuous Quality Evaluation (MMDSCQE). Subsequently, the new syntax was approved by the ISO/IEC MPEG-4 committee and resulted in a new profile know as the Error Resilient Simple Scalable Profile (ER-SSP). It is shown that minor modifications are required in the Header Extension tool to synchronize the decoding process between the enhancement and the base layer. Thus it is shown that base layer error resilience tools are equally applicable to the enhancement layer with nominal syntax changes.

Keywords: Error resilient video coding and MPEG-4 scalable video coding.

\footnotetext{
${ }^{1}$ Author for correspondence
} 


\section{Introduction}

The need for reliable and resilient scalable video transmissions over wireless networks is becoming a necessity especially with the advent of new mobile communication technologies.

Reliable transmission of video is realized by following a number of preventative and corrective measures. For example, the MPEG-4 video specification [1] provides for preventative measures by standardizing a variety of error resilience tools such as Video Packet (VP) structures, Header Extensions (HE), Data Partitioning (DP) and Reversible Variable Length Codes (RVLCs).

Likewise, with differentiated quality of services, scalable video coding in MPEG-4 can be employed to increase the probability of transmitting the most vital visual information to the receiver. Scalable video can also minimize the corruption of visual information by matching different or varying network bandwidths.

Corrective measures, on the other hand, include, but are not limited to, error correction and concealment which are outside the scope of this paper. Interested readers can refer to an in-depth treatment of interframe error concealment as reported by the author in $[2,3]$.

The error resiliency tools of MPEG-4 are part of the Simple, Core, Main, Advanced Simple and FGS streaming profiles. These tools however, were not supported by the Simple Scalable Profile (SSP) syntax. Hence the use of SSP was limited to error-free communications that excluded mobile communications.

However scalable video can be very useful in many other applications. For instance, matching different or varying network bandwidths, video multicast to heterogeneous end systems and more importantly, providing different subjective quality of video content to subscribers of a given video service depending on their network tariffs and viewing preferences. In this case, loss of visual data in the enhancement layer could represent a significant revenue impact for service providers if end-users lose confidence with the reliability of the service. Simulations of MPEG-4 Simple Scalable Profile (SSP) video communications under error conditions that typically occur in GPRS and CDMA 2000 networks 
have revealed that significant subjective quality penalties are incurred by the prohibition of use of error resiliency tools in the enhancement layers.

To alleviate this problem, we report on syntax friendly approaches to error resiliency in the Simple Scalable Profile. Such solutions are syntax compliant but affect coding efficiency and have many other limitations as elaborated upon in the paper. Consequently, the paper reports on modifications to the SSP syntax as originally proposed in $[4,5]$. The proposed syntax changes resulted in a new profile under the name Error Resilient SSP profile [6]. The new profile enables the deployment of scalable coding in error prone mobile multimedia communication environments whilst preventing the degradations and limitations of the reported syntax friendly approaches.

It is worth reiterating that MPEG-4 incorporates error resilience tools in the enhancement layers of the Fine Granularity Scalability (FGS) as proposed in $[7,8]$. However, the SSP profile imposes a number of additional challenges as follows. Firstly, FGS codec has an inherent layered bitstream structure that facilitates resynchronization at the beginning of each Bit Plane (BP) within a coded enhancement VOP. This self-synchronization property is absent from the Simple Scalable Profile. Secondly, in FGS, as opposed to Temporal Fine Granularity Scalability (TFGS), corrupted VOPs can immediately recover from transmission errors at the beginning of the next VOP. This is because the source of prediction is restricted to the base layer VOPs without motion compensation. Hence errors will not propagate in the temporal domain. Lastly, B-VOPs in TFGS have a fixed 'fgs_ref_select_code' of '11' i.e. the forward and backward reference VOPs are fixed and known a priori. In SSP on the other hand, each of $\mathrm{P}$ and $\mathrm{B}$ VOPs have four different prediction combinations and hence four different values for 'ref_select_code'. As such, under transmission errors, the decoder must ensure that it is reconstructing the enhancement VOP from the correct reference VOPs whilst ensuring correct display order of both video layers. The paper elaborates upon such challenges and proposes a number of solutions as well. 


\section{The need for error resiliency in the SSP.}

In comparison to error resiliency in the Simple Profile, the Simple Scalable Profile imposes a higher resiliency challenge. In the latter profile the decoder must remain in sync with two separate bit streams potentially with re-ordered VOPs. The decoder must also reconstruct the enhancement VOPs from the correct reference sources and display them in the correct order.

Therefore enhancement layers in the SSP profile have to cater for two categories of error resiliency; intra-layer and inter-layer resiliency. The need for the two categories is discussed in the following sections.

\subsection{Intra-layer resiliency}

Similar to the SP profile, in intra-layer resiliency the decoder must be able to recover from errors affecting VOPs' motion parameters and/or texture data. The trend in this case is to localise the errors such that they do not propagate in the spatial and/or the temporal domain. Spatial error propagation is due to the nature of variable length coding. Likewise temporal propagation is due to the nature of interframe coding.

To alleviate this problem, three error resiliency tools are defined in the MPEG-4 visual standard. Resynchronisation markers are used to localise errors within video packets [9-11]. Data partitioning provides an additional resynchronisation marker (i.e. motion marker) that separates the motion parameters from texture data and therefore provides higher interframe error concealment quality. Lastly, errors in the texture data coded after the motion marker can be further localised by the Reversible Variable Length Codes [12]. For the purpose of intra-layer resiliency, these tools are equally applicable to the enhancement layer.

The importance and the performance of these error resiliency tools are well established in the Simple Profile hence a brief description of their use in the enhancement layer is elaborated upon in the following section.

\subsubsection{Video Packet Headers}


This error resiliency technique contains two useful tools, resynchronisation markers and Header Extension (HE) tools. This section deals with the resynchronisation markers. The HE is dealt with separately in Section 8.1.

When an erroneous variable length code is received, the decoding process loses its synchronisation with the underlying bit stream until a resynchronisation marker is encountered. In the case of enhancement layers this is the start code of the next VOP. The lack of error localisation in this case will cause the rest of the underlying VOP to be concealed regardless of whether or not further errors are encountered.

The effect of this concealment depends on the employed scalability mode. In spatial scalability where the enhancement layers increase the resolution or the quality of the base layer, concealment may result in VOPs enhanced at the top but not at the bottom of an image frame. In temporal scalability where the enhancement VOPs do not coincide temporally with the base layer, the concealment results in displaced macroblocks typically comprising large areas of the VOP. As a side effect, and depending on the interframe coding modes this displacement shall propagate into future decoded VOPs.

\subsubsection{Data Partitioning}

Although Video Packet Headers are a useful tool for localising errors nevertheless corrupted visual data is concealed by means of estimating the values of lost motion vectors and motion compensating previous VOPs. Typically, the lost motion vectors are estimated from either the surrounding or the co-located macroblocks in previous VOPs. It follows that these motion vectors are not accurate and they usually cause the displacement of concealed macroblocks. As such, the MPEG-4 visual standard embraced an error resilience tool called Data Partitioning (DP) that separates the motion parameters from the texture information i.e. DCT coefficients. This separation is performed on a Video Packet basis where an additional resynchronisation marker, called Motion Marker, separates the motion parameters from the DCT coefficients. If an error occurs in the DCT coefficients part then the original motion vectors, as opposed 
to the estimated motion vectors, are employed to conceal the rest of the Video Packet.

The DP tool is applicable to the enhancement layers are well. One exception is when the enhancement layer VOPs are predicted from the base without motioncompensation, this applies to P-VOPs in the SNR and Spatial scalability modes. In this case there are no motion vectors to separate from the texture coefficients hence a special arrangement is needed as discussed in the syntax changes of Section 8.

\subsubsection{Reversible Variable Length Code (RVLC)}

Having separated the DCT coefficients from the motion vectors, errors in the DCT coefficients part can be concealed by means of motion compensation using the correctly received motion vectors. The correctly received DCT coefficients up to the location of errors can be used as well. The discarded coefficients might cause visual distortions.

This degradation can be overcome by reducing the number of concealed macroblocks through the use of RVLCs. According to the MPEG-4 specification, RVLCs can only be used in combination with the DP tool. In this case if an illegal or invalid VLC code is encountered, the decoder starts the backward decoding from the end of the Video Packet until it encounters another invalid VLC code. Note here that the two-encountered errors might belong to two different macroblocks.

As mentioned previously, note that the importance of the DP and RVLC tools vary from one scalability mode to another. For instance in Spatial and SNR scalabilities, P-VOPs are always reconstructed without motion compensation i.e. with nil motion vectors. Separating motion parameters from texture data in this case does not make much sense unless RVLC is employed. This is because corrupted macroblocks are often concealed by copying them from the co-located ones of the base layer VOP without motion compensation. On the other hand, for all other cases where the macroblocks are motion compensated for prediction, data partitioning is a useful tool indeed regardless of the use of RVLCs. 


\section{2 Inter-layer resiliency}

In this category the decoder must be able to maintain synchronisation between the decoding of two video layers. Loss of inter-layer synchronisation here can be caused by VOP header errors in the enhancement layer. For instance, if VOP reordering is employed for bi-directional prediction then the corruption of VOP timestamps might lead to out-of-order decoding and display. Likewise, such errors can also mislead the decoder into selecting false reference sources for the reconstruction of the underlying enhancement VOP.

The MPEG-4 visual specification defines a useful tool here called Header Extension which is signalled by the Header Extension Code (HEC) in the Video Packet header. The HE replicates vital VOP header information to facilitate independent decoding of Video Packets. Hence if a VOP header is received in error then once the HE is reached, correct and synchronised decoding is resumed.

In the Simple Profile a predicted VOP is, by default, reconstructed from the immediate previous VOP. Hence a corrupted VOP timestamp may alter the display time, nevertheless the decoded VOPs will still be reconstructed from the correct reference sources and displayed in the correct order.

On the other hand, a predicted VOP in the Simple Scalable Profile is not necessarily reconstructed from the immediate previous VOP. Hence a corrupted VOP timestamp may alter both the display time and the decoding order. This is particularly true in temporal scalability where the VOPs of the two layers do not coincide temporally. Here the decoder must decide on which VOP to decode next, i.e. whether it belongs to the base or the enhancement layer. This decision is entirely dependent upon the VOPs timestamps. Getting this wrong results in out-of-order decoding and displaying. Consequently, the out-of-order decoding will cause the enhancement VOPs to be reconstructed from false references and the error starts to accumulate until it corrupts the whole enhancement layer.

Fortunately the Header Extension of Video Packet Headers can be utilised to alleviate this deficiency by replicating the timestamp of the respective VOP. Once decoded the correct values can be used to resume correct and synchronised 
decoding. The effect of such an arrangement on decoding quality is illustrated in the experimental results section.

\subsection{Experimental results:}

This section illustrates the decoding degradations caused by the absence of error resiliency tools in the enhancement layers of the SSP profile.

To illustrate the importance of the synchronization marker in the enhancement layers, the "Soccer" sequence is coded with temporal scalability. Each layer is coded at 20kbit/s. Resynchronisation markers are injected at a periodic rate of 800 bits. The coded enhancement sequence was then presented to a GPRS network simulator ${ }^{2}[13]$ with a Frame Erasure Rate (FER) of $3 \%$ and Residual Bit Error Rate (RBER) of $0.047 \%$.

Figure 1 plots the PSNR results of the decoded sequences. As the figure shows the absence of Video Packet Headers caused spatial error propagations hence the PSNR drop of up to $7 \mathrm{~dB}$. The figure also shows improvement in decoding quality when the resynchronisation markers are used in the enhancement layer.

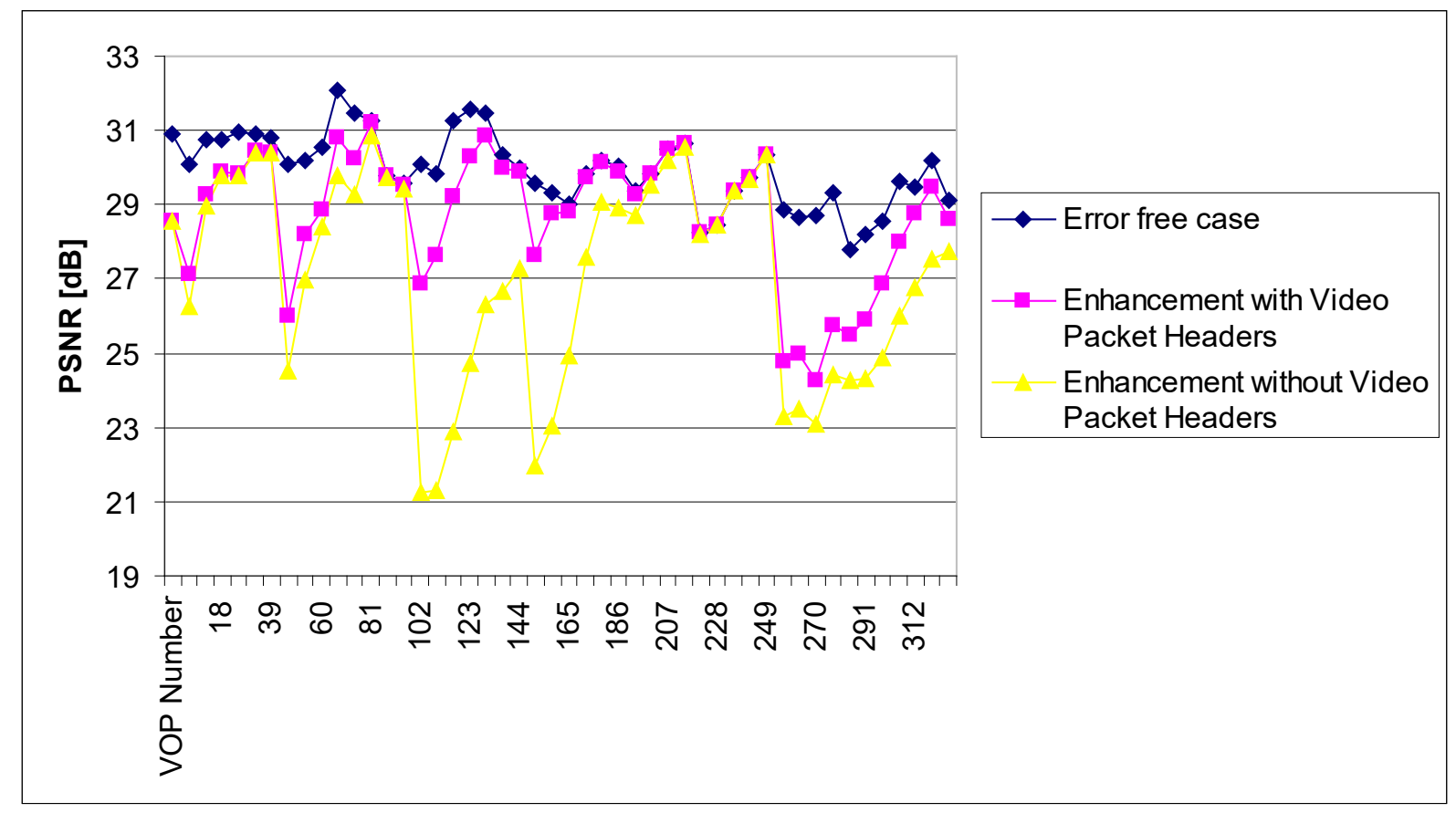

${ }^{2}$ Coding Scheme 4 (CS-4), Typical Urban environments with 4 time slots are assumed throughout the simulations. The simulator is developed at Motorola, Swindon (UK) [8-]. 
Figure 1. Objective degradations associated with the absence of resync markers.

Since the importance of incorporating the error resiliency tools in the enhancement layers of the SSP profile is now established we move on to present two approaches for this resiliency; with and without bitstream syntax modifications.

\section{A syntax friendly approach to error resiliency}

Since the existing bitstream syntax and profile definition prohibited the use of error resiliency tools in the enhancement layer of the SSP, the purpose of this section is to reveal alternative solutions for achieving error resiliency whilst adhering to the syntax of the SSP. The syntax friendly solutions give an excellent insight into the relevant syntax and semantics of the SP and the SSP profiles.

\subsection{Alternative solutions to resynchronisation markers}

In MPEG-4, the resynchronization marker is distinguished from all other VLC codes and consists of 16 zeros followed by a number of ones depending on the VOP type and its fcode value. When encountered the decoder resets its predictors including the macroblock address, quant_scale and motion vectors. The absolute value of the macroblock address and quant_scale are present just after the resynchronization marker. The motion vector predictors are set to nil. Therefore to introduce resynchronization markers into the enhancement layers without breaking the existing bit stream syntax, a number of problems are addressed as follows:

a. Resynchronization markers have to be introduced in the enhancement bitstream, we refer to them as Faked Resynchronization Markers (FRM). These markers should not break existing compliant decoders. 
b. Once a FRM is decoded, the current absolute macroblock address and absolute quant_scale should be established. Again these values must pass through compliant decoders in a transparent manner.

c. If a modified decoder resets it motion vector predictors upon encountering a FRM then it will become incompliant. Hence the proposed solution introduces a constraint set of coding configurations that overcomes this compliancy issue.

The first two points relating to faking resynchronization markers can be solved using STUFFING BITS. Before presenting the solution, stuffing bits are elaborated upon. Stuffing bits are used to increase the number of generated bits per VOP. The rationale is to prevent the decoder's buffer from underflow. These bits are also used to ensure the byte-alignment of start codes. Likewise if fixed interval synchronization is employed in the base layer then stuffing bits may be used to ensure precise interval lengths, in terms of bits that is. However the last two usages are not related to the proposed solution, therefore they will not be discussed any further.

Common to the usage of stuffing bits is the discarding of these bits during the decoding process; hence they pass through the decoder in a transparent manner.

Stuffing bits may be found at macroblock headers, namely if the 'mcbpc' (MB type and coding block pattern for chrominance) has the value of '0000 00001 ' then this is interpreted by the decoder as stuffing bits. Once encountered and ignored by the decoder, the macroblock decoding loop is resumed.

One method of achieving Faked Resynchronization Markers is realized by inserting more than one group of stuffing bits at the beginning of predetermined macroblock rows. For instance, FRM can be injected at a fixed rate of two MB rows. Figure 2 illustrates this for a QCIF image resulting in the use of three FRMs. 


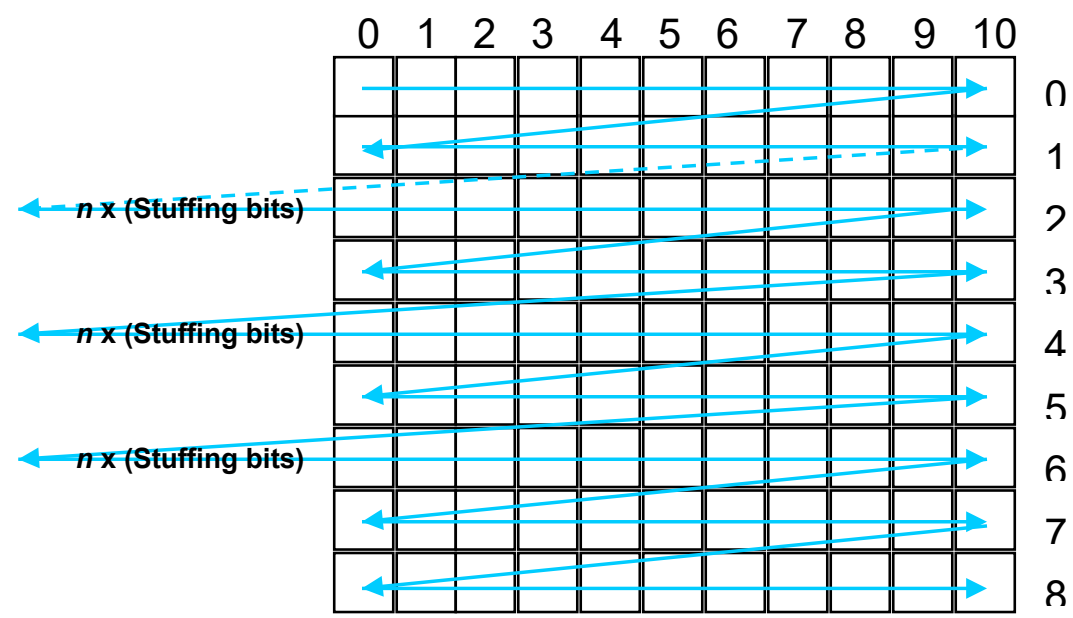

Figure 2. Possible positioning of FRM in a QCIF picture.

The directed lines in the figure refer to the scanning or transmission order of the macroblocks and FRM. In this example it is prearranged at the codec level that the FRM are injected with a spacing of two MB rows. This can be signaled, alongside the number of stuffing bits groups employed, to the decoder in the 'user data' field of the VOL header. At a system level however, such arrangements can be signaled prior to streaming the bit streams.

Additionally, similar to the true resynchronization markers, this work proposes to byte-align the FRMs. However, since the beginnings of MB rows are not guaranteed to be byte aligned, special coding arrangements should take place as outlined in this section.

Recall from video coding basics that byte-alignment for resync markers including start codes have a number of benefits. First, if sync is lost with the underlying bitstream, the decoder shall search for the next resync marker at byte rather than bit locations, hence simplifying the decoding process. Second, under bit error conditions, searching for the next resync marker on byte basis implies lower probability of resync marker emulation. Finally, byte-alignment can also serve as an error detection method should a resync marker or an emulated one is found in a non-byte-aligned location. 
This section proposes the introduction of noise by adding a number of $A C$ coefficients in the high frequency band of the last DCT block preceding a FRM. The procedure is outlined in the following flowchart diagram.

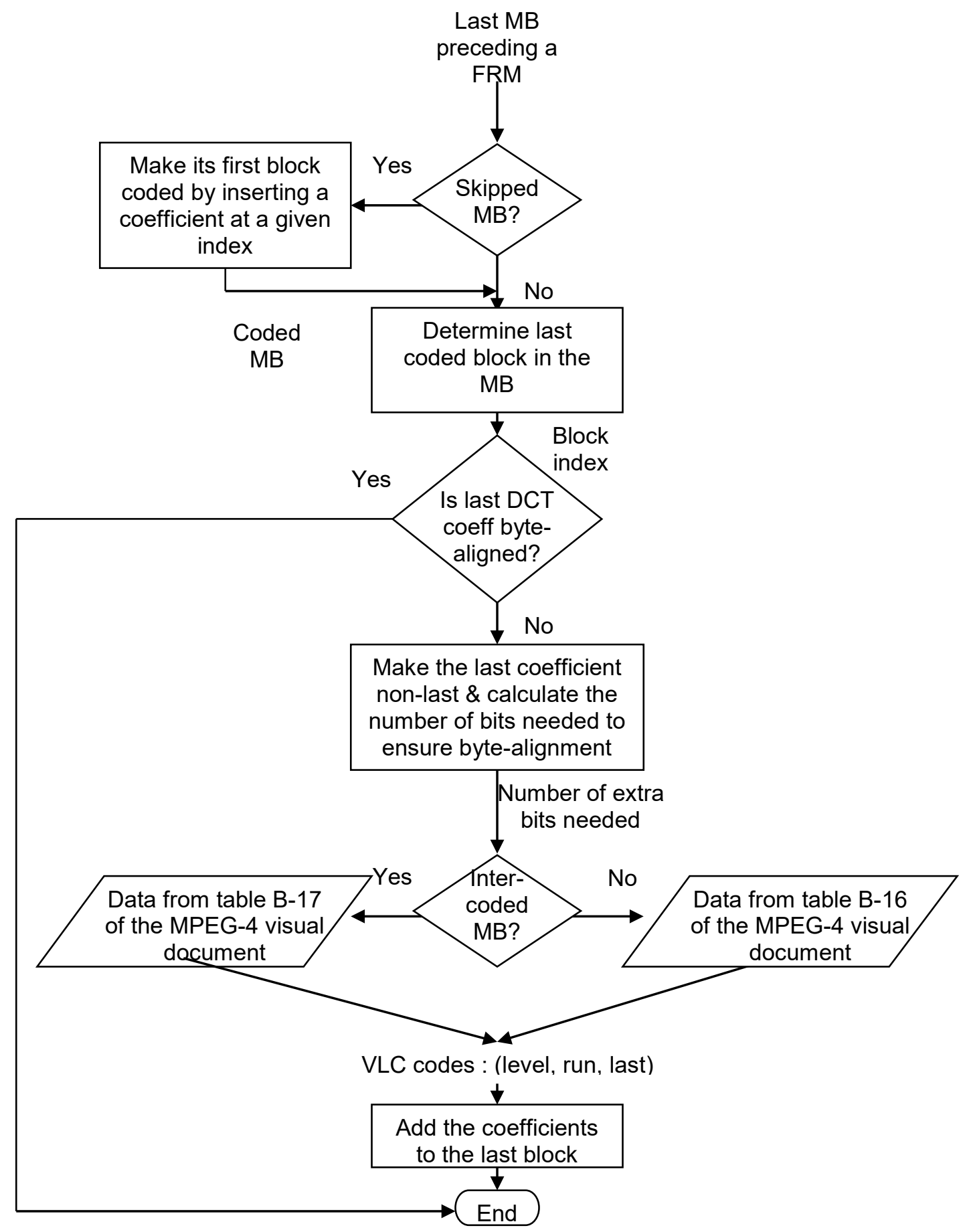

Figure 3. Flowchart for ensuring byte-alignment of FRMs. 
In short, if the last DCT coefficient is not byte-aligned then that coefficient is made non-last and put to the bitstream. Consequently, the decoder calculates the number of bits needed to complete a byte and outputs some pre-defined VLC codes from either Table B-16 or B-17. Appendix A gives one possible set of VLC codes for the case of inter-coded macroblocks.

\subsubsection{Resetting the Motion Vector Predictors.}

In the presence of bit errors or packet losses, a typical compliant decoder starts discarding bits until it encounters a resynchronization marker. The very fact that corrupted bits, including motion vectors, are discarded implies that motion vector prediction must be reset each time a resynchronization marker is encountered. In fact MPEG-4 employs a Video Packet structure. The boundaries of which are treated as if they were a VOP boundaries hence predictors from outside the Video Packet are prohibited.

For the FRM solution proposed above, the motion vector predictors have to be reset in a similar manner. The only danger here is that MV resetting without true resynchronization markers might render a decoder incompliant. This problem can be alleviated in one of two approaches. First, in Spatial and SNR scalabilities the encoder can be configured such that the prediction of the enhancement layer is restricted to the base layer without motion compensation. As such no motion vectors are transmitted in the bitstream and therefore resetting the motion vector predictors becomes irrelevant altogether.

On the other hand, in temporal scalability restricting the motion vector value to nil will result in poor coding quality depending on the amount of motion between successive VOPs. Hence prior to solving this problem we start by reviewing the motion vector prediction process as defined in MPEG-4. 
Figure 4 shows that every motion vector is predicted from three surrounding values numbered MV1, MV2 and MV3.

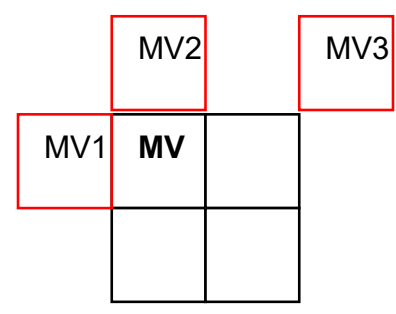

(a)

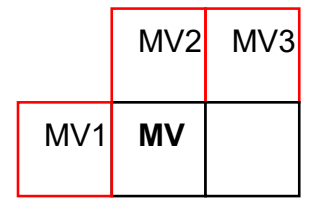

(c)

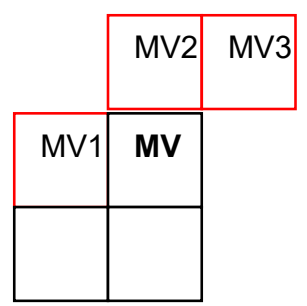

(b)

\begin{tabular}{|l|l|}
\hline MV2 & MV3 \\
\hline MV1 & MV \\
\hline
\end{tabular}

(d)

Figure 4. Location of motion vector predictors for each of the four luminance blocks.

When a resynchronization marker is encountered at a beginning of a MB row, a compliant decoder will set the MV2 and MV3 of part (a) and (b) of the above figure to an invalid status. Note that in this case the motion vector predictors in parts (c) and (d) are unaffected.

This work proposes to set the values of MV2 and MV3 of parts (a) and (b) to nil in the first place. That is by enforcing this value at the encoder. Hence when a FRM is encountered resetting these motion vector predictors will not make a difference. As such, in compliant decoders which will not reset when encountering a FRM will still be capable of correctly decoding the underlying VOP.

However, there is one string attached to this solution, MPEG-4 specifies that if two and only two motion vector predictors are invalid then they are set to the value of the third predictor. So in our example MV2 and MV3 of parts (a) and (b) are set to the value of MV1. For instance if the value of MV1 is $(2,3)$ and MV2, MV3 are invalid then by resetting them, the value $(2,3)$ will be the median motion vector predictor. Whereas in compliant decoders where MV2 and MV3 are not 
reset but rather have the value $(0,0)$, the median $\mathrm{MV}$ predictor in this case will be $(0,0)$ which differs from the FRM case and hence will not be correctly decoded.

To solve this problem it is essential to ensure that the value of MV1 in parts (a) and (b) are also set to zero during the encoding process. Hence the value of the MV predictor in this case is $(0,0)$ regardless of whether or not the underlying decoder reset its MV predictors. The bottom line is as follows: at the encoder, restrict the predictors to within the boundaries of the faked Video Packet in the first place.

This solution is summarized in Figure 5 below.

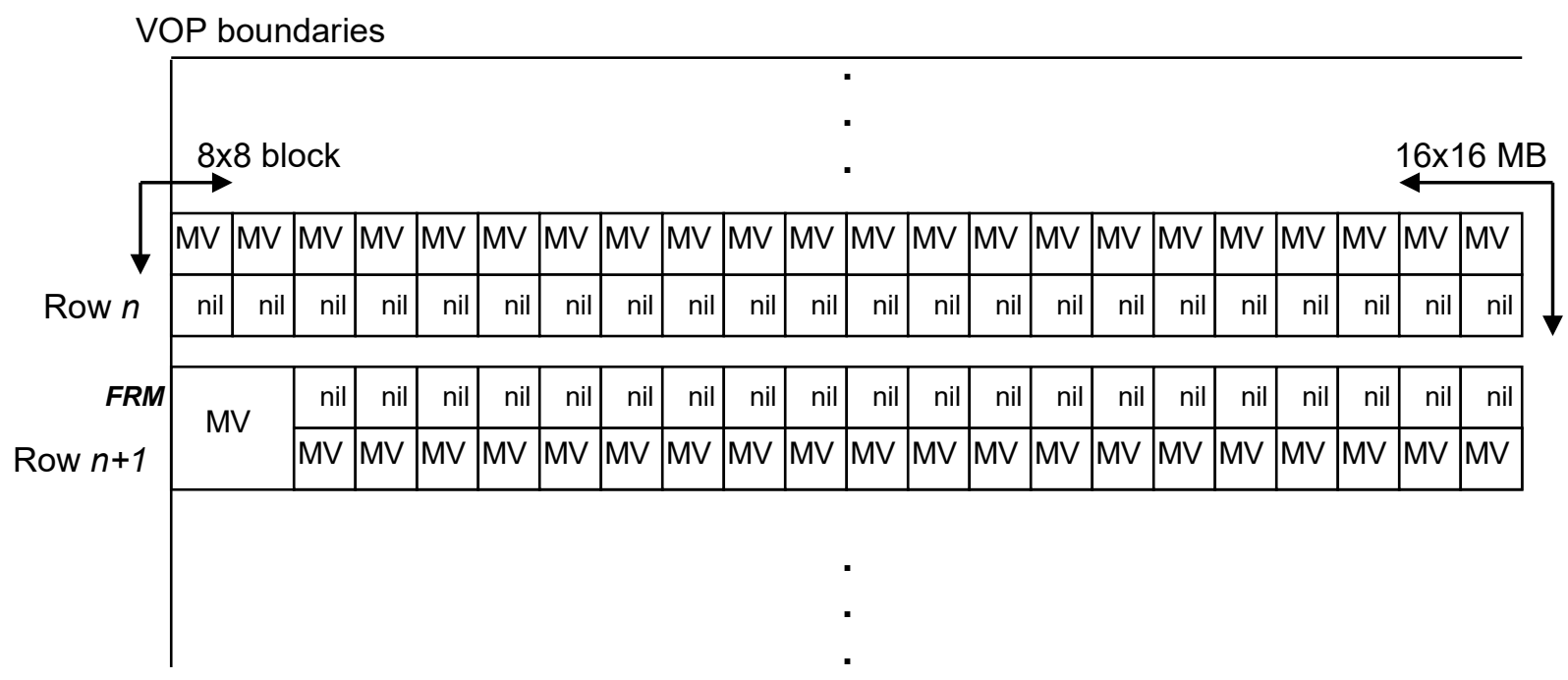

Figure 5. Macroblock coding modes for both the FRM MB row and its immediate previous row.

As shown in the figure, the MB row preceding a FRM must have special motion compensation modes. That is, the values of its motion vectors (for the lower $8 \times 8$ blocks at least) are forced to nil during the encoding. Likewise, the upper $8 \times 8$ blocks of a FRM MB row shall also have the value of nil. Note here that the encoder is free to choose among three coding modes; intracoding, inter coding without motion compensation or 4 motion vector (4MV) per MB provided that either the lower or the upper MVs are set to nil as explained above.

Note that the first MB of the FRM row should not be set to $4 \mathrm{MV}$ mode since this will affect resetting the quantizer value as discussed in later sections. 
Clearly any interference with the encoder's motion compensation decisions will affect the coding efficiency, this is discussed in Section 6 below.

\subsubsection{Reinitialising the quantization index predictor}

Likewise when a FRM is decoded the quantization index or 'quant_value' has to be reinitialised. In the base layer base case, the absolute 'quant_value' is available in the Video Packet Header. For our syntax friendly approach, the quant_value can be reinitialised by copying the VOP's quantizer decoded from the VOP header known as 'vop_quant'. Again special arrangements are sought here to ensure that this passes through compliant decoders without being noticed.

This can be arranged at the encoder side, the trick here is to ensure that the quant_value of a macroblock following a FRM is always equal to the 'vop_quant'. In the MPEG-4 specification, the quantization index is encoded as an incremental value using the field 'dquant' and therefore the encoder should ensure that the following equality holds after each FRM:

$$
\text { dquant }=\text { vop_quant }- \text { Current } Q \text {. }
$$

Where CurrentQ is the quantizer of the first MB following a FRM. Bear in mind here that the MPEG-4 specification limits 'dquant' to the range $[-2,2]$ hence the encoder has to make sure that this range is met by planning it a number of macroblocks in advance. An algorithm to solve this problem is devised and illustrated in Appendix B.

\subsubsection{Resetting the AC predictors}

In Intra-coded macroblocks, the first AC row or the first AC column of each block can be predicted from either the preceding or the above block. The AC prediction is employed provided that the 'ac_pred_flag' of the macroblock header is set to 1 . 
Since the borders of video packets are treated as VOP borders, the AC coefficient prediction is restricted to within the borders of the Video Packet. For our purpose, the AC predictors have to be reset each time a FRM is decoded.

Basically if the 'ac_pred_flag' is set to 0 each time a block belonging to a given macroblock is predicted from outside the Video Packet border then the problem is solved. As such, resetting the AC predictors on encountering a FRM will have no effect because the AC prediction was confined to within the Video Packet in the first place.

\subsubsection{Resetting the DC predictor}

Similarly, the DC of blocks belonging to Intra-coded macroblocks can be predicted from either the preceding or the above block. If any of the prediction source blocks are outside the VOP's border or the Video Packet's border then that DC predictor is set to 1024 .

Unlike AC prediction, the DC prediction is activated at all times and there is no flag in the headers to deactivate it. Hence the prediction cannot be confined to within the Video Packet border as the case for AC prediction.

Recall that FRMs are located at the beginning of predefined MB rows hence the problem with $\mathrm{DC}$ prediction is confined to employing the above MB for prediction. Otherwise the prediction is confined within the faked video packet. Therefore one way around this drawback is to prevent intra coded macroblocks from appearing at the same MB row of a FRM. Considering that FRMs appear at most once every 2 rows of MBs, it follows that DC prediction from the above MBs will not appear unless the above row of MBs belongs to the same faked video packet.

\section{Alternative solution to the Header Extension (HE)}

Header Extensions are available in Video Packet Headers provided that the Header Extension Code (HEC) flag is set in the VP header.

A possible layout of a base layer VOP that employs the Video Packet structure is illustrated in Figure 6. 


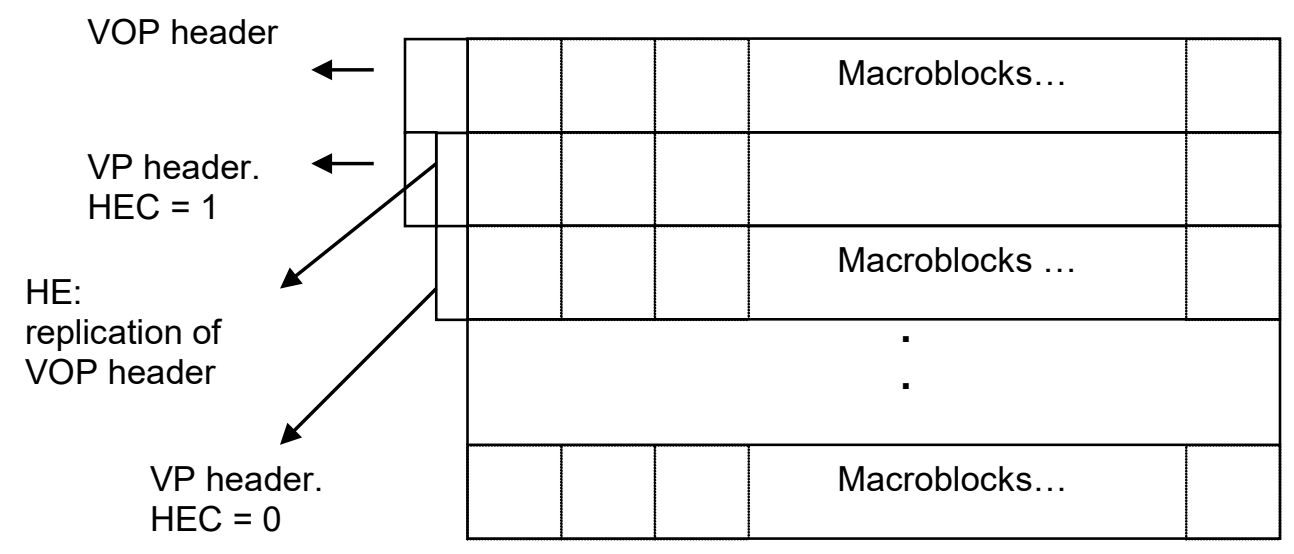

Figure 6. Possible VOP layout with a Video Packet structure.

As illustrated in the figure, a base layer VOP may have a number of VPs, each of which might or might not have a HE according to the value of HEC. Usually in MPEG-4 SP implementations only one HEC is set and therefore only one HE is transmitted. The rationale behind this is to keep the number of overhead bits to a minimum.

The solution proposed in this section is to utilize one of the HEs of a base layer VOP to replicate the header information of a preceding enhancement layer VOP. This arrangement shall work smoothly given that the base layer VOPs are encoded and consequently decoded one VOP in advance. This is because the enhancement layer VOPs might employ temporally successive VOPs of the base layer as a source of prediction hence these VOPs (from the base layer) are encoded and reconstructed prior to encoding the enhancement layer VOP. Likewise at the decoder, the base layer VOP is decoded one VOP is advance to ensure that the enhancement layer VOP can be reconstructed correctly. This difference between the encoding and displaying order is illustrated in an example in Figure 7. 


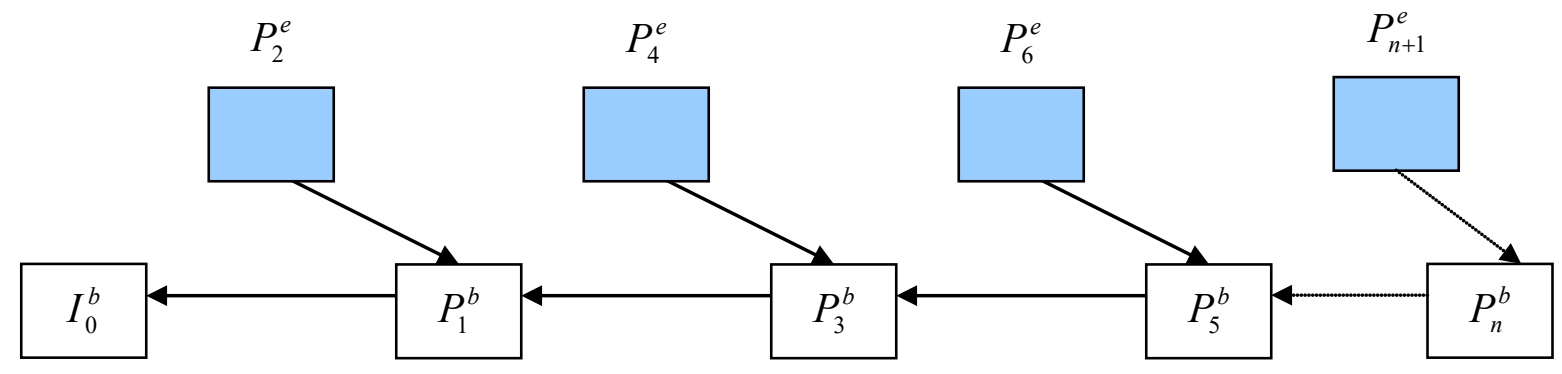

Enhancement layer VOPs.

Base layer VOPs.

Figure 7. The difference between encoding and displaying order in the MPEG-4 SSP.

In the figure, each VOP type has a subscript to indicate its encoding number and a superscript to indicate the layer it belongs to i.e. base or enhancement. The VOPs are shown in displaying order and are numbered in encoding order. This arrangement holds for both encoding and decoding regardless of the directionality of the enhancement layer prediction.

Likewise, once a VOP is coded, the rate control decides on the number of VOPs to skip in order to meet a given bitrate target. Therefore the timestamp of a VOP is known prior to encoding it. Additionally, since the 'vop_quant' of a given VOP is calculated based on coding information gathered from the previous VOP then the 'vop_quant' will also be known prior to encoding a VOP. For instance when $P_{3}^{b}$ from Figure 7 is encoded, the timestamp and the 'vop_quant' of $P_{4}^{e}$ are know beforehand, hence this information can be replicated in one of the HEs of the base layer VOP. The encoder can then move on to code the enhancement layer VOP, $P_{4}^{e}$ in this case. This solution is illustrated in Figure 8. 


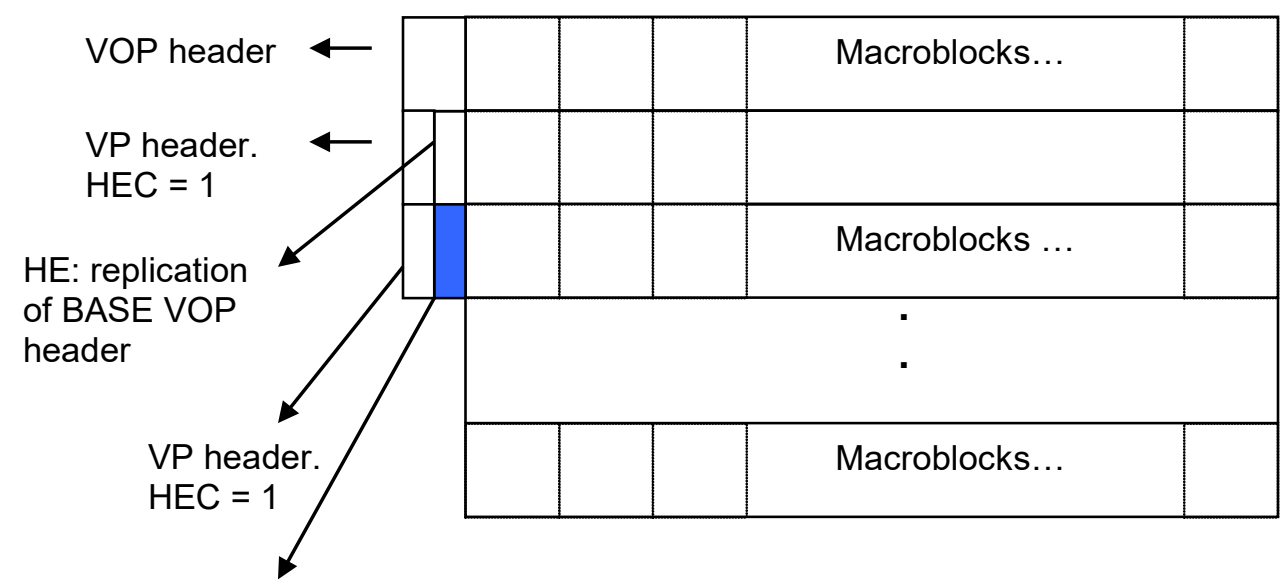

HE: replication of ENH VOP header

Figure 8. The proposed solution. Replicate some of the enhancement VOP header info in a HE of the base layer VOP.

In the figure, the HE of the first VP is utilized to replicate the header information of the underlying base layer VOP. In contrast the HE of the successive VP is utilized to replicate the header information of the preceding (in display order) enhancement layer VOP.

Note that the action taken upon decoding a Header Extension, which is error recovery, is an informative rather than a normative part of the MPEG-4 visual specification. Therefore, manipulating the semantics of such fields should not be a violation to the MPEG-4 specification. More specifically, storing the header information of an enhancement VOP in the HE of a base VOP is not believed to be non-compliant. However under error recovery conditions, compliant decoder implementations might break because of such an arrangement.

\section{Experimental results}

Common to all of the experiments to follow the coded bitstreams are transmitted over GPRS channel conditions using a GPRS simulator [13]. Each bitstream is coded at around $21 \mathrm{kbps}$. The generated frame-erasure and bit error rates are given for each experiment.

Furthermore, since the introduction of FRM in SNR/Spatial scalability is a subset of introducing it in Temporal scalability, the following results focus on Temporal 
scalability. Recall that in P-VOPs of the SNR/Spatial scalability, the MPEG-4 specification does not allow the use of motion vectors in the enhancement layer hence the introduction of FRM is by far less complex.

Two coding modes are considered with 'ref_select_code' ='00' (referred to as Case A) and 'ref_select_code'='01' or '10' (referred to as Case B) as shown in Figure 9 below. The 'ref_select_code' field specifies the prediction references for $P$ and $B$ VOPs in enhancement layer.

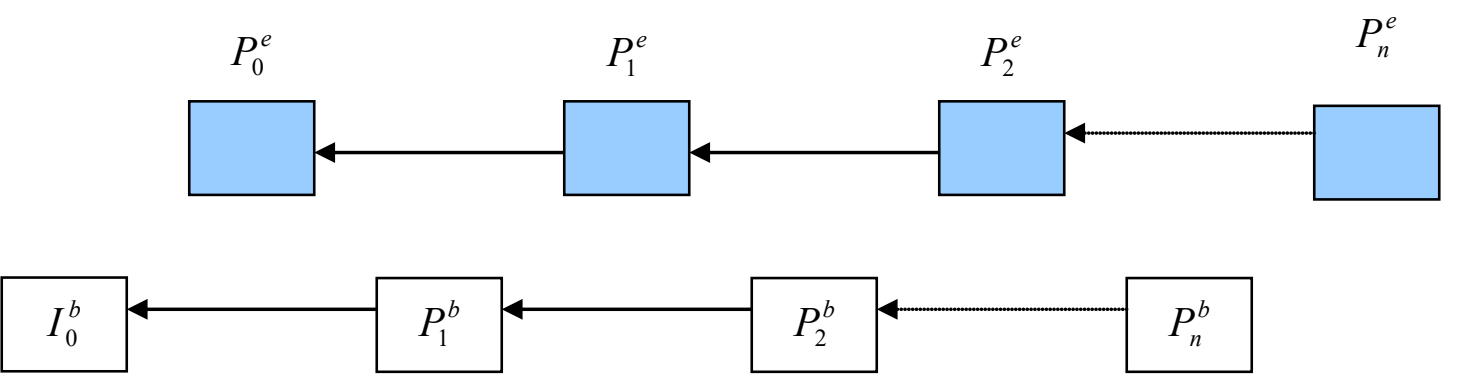

(a) Case A: ref_select_code = '00'

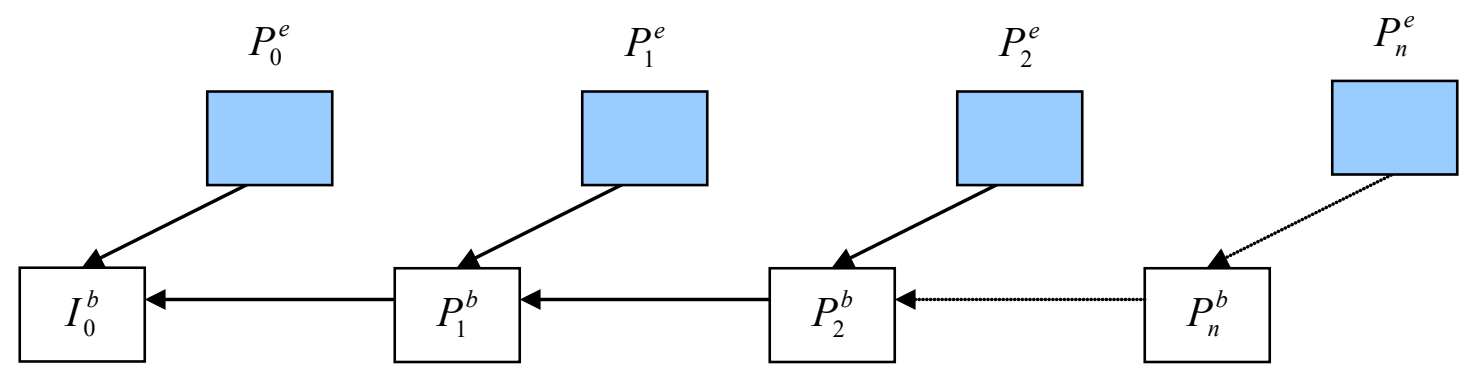

OR:

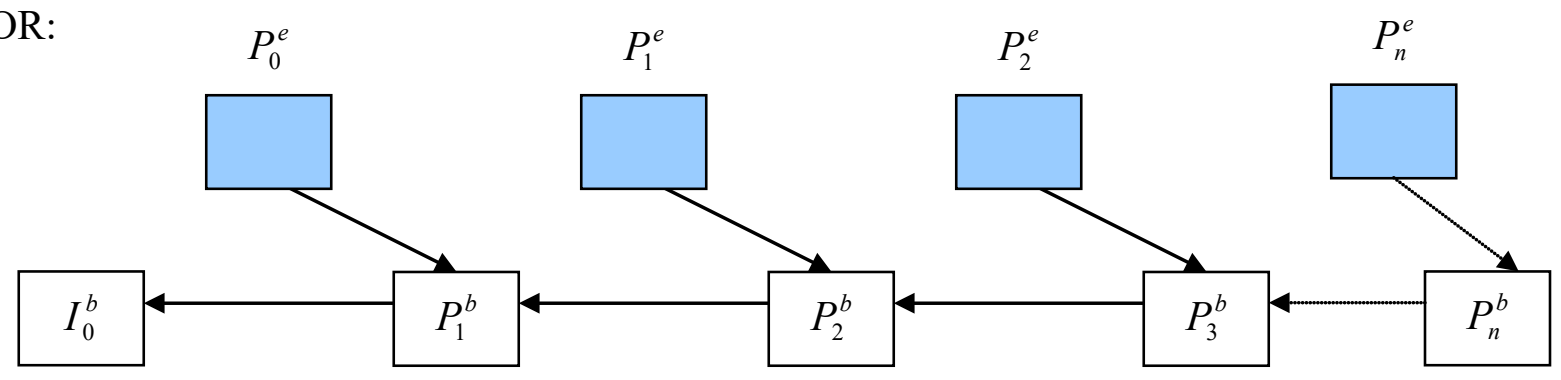

(b) Case A: ref_select_code = '01' and '10' respectively

Enhancement layer stream.

Base layer stream.

Figure 9. Coding modes considered in the experimental results. 


\subsection{Case A results:}

In this scenario, since the enhancement VOPs are predicted from each other it follows that errors will propagate in the temporal domain and hence provide a better insight into the effectiveness of FRMs.

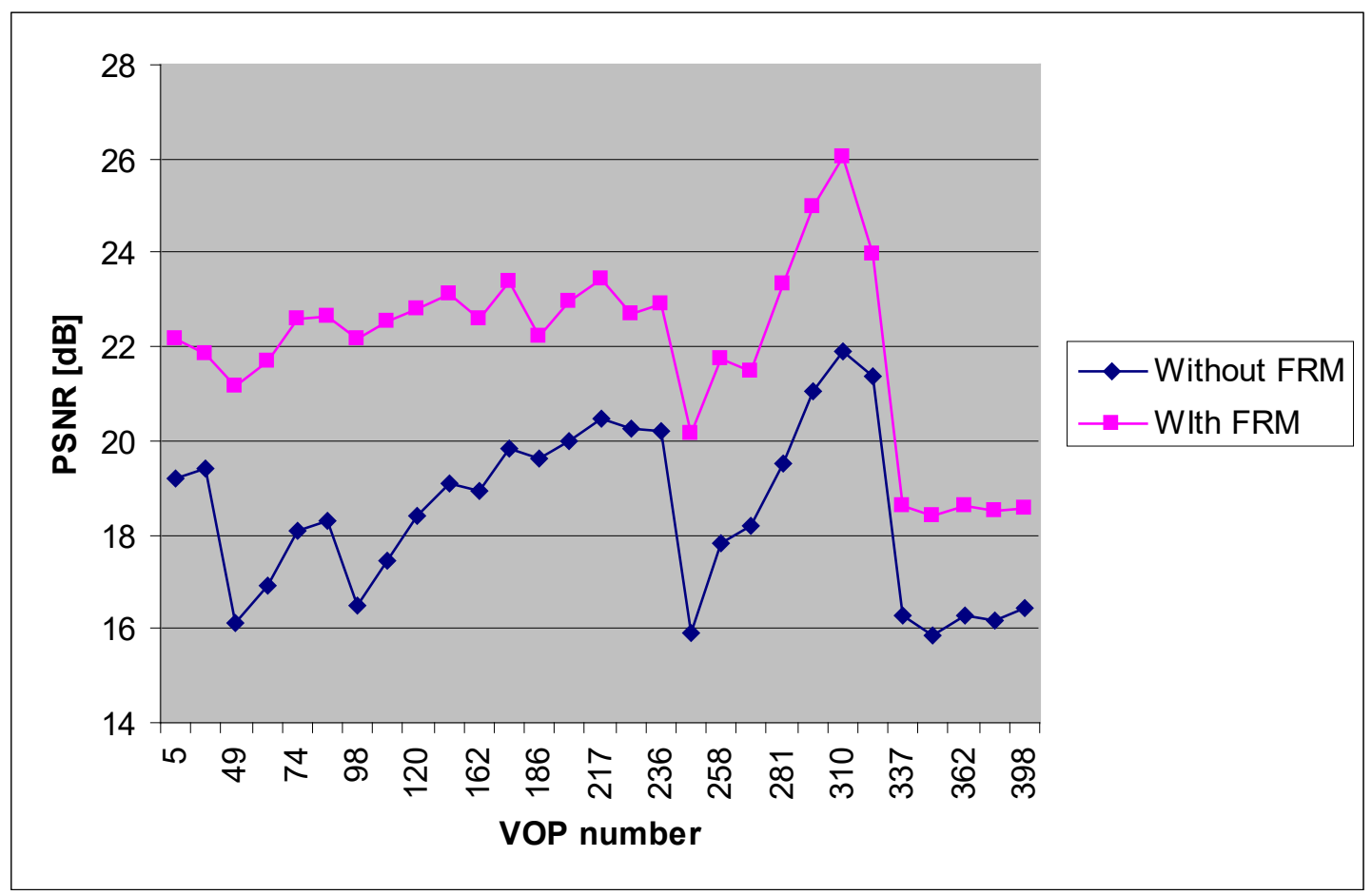

(a) FOREMAN sequence. Frame Erasure Rate $=3.23 \%$, Residual Bit Error Rate $=0.06 \%$. 


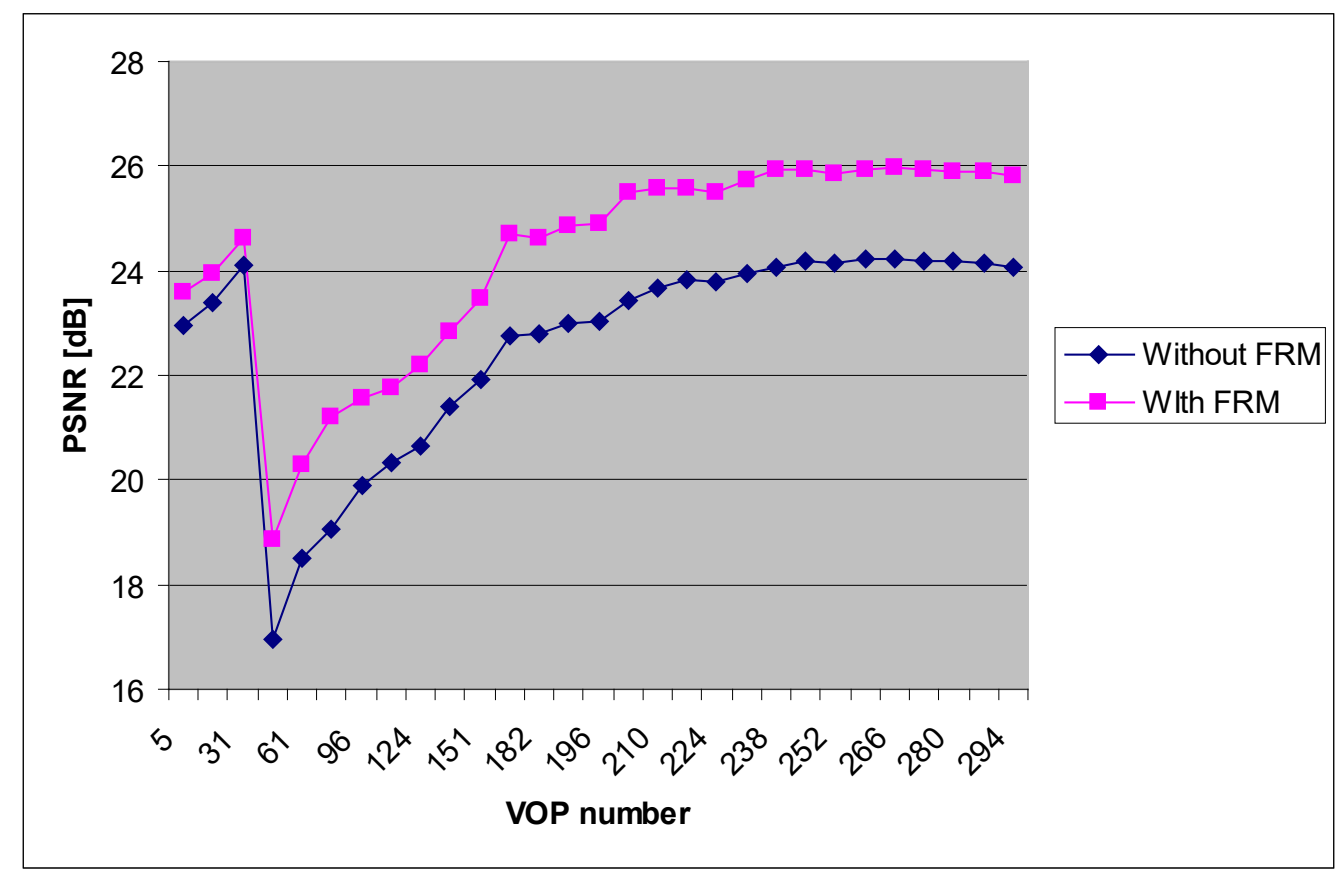

(b) TABLE sequence. Frame Erasure Rate $=2.8 \%$, Residual Bit Error Rate $=$ $0.046 \%$.

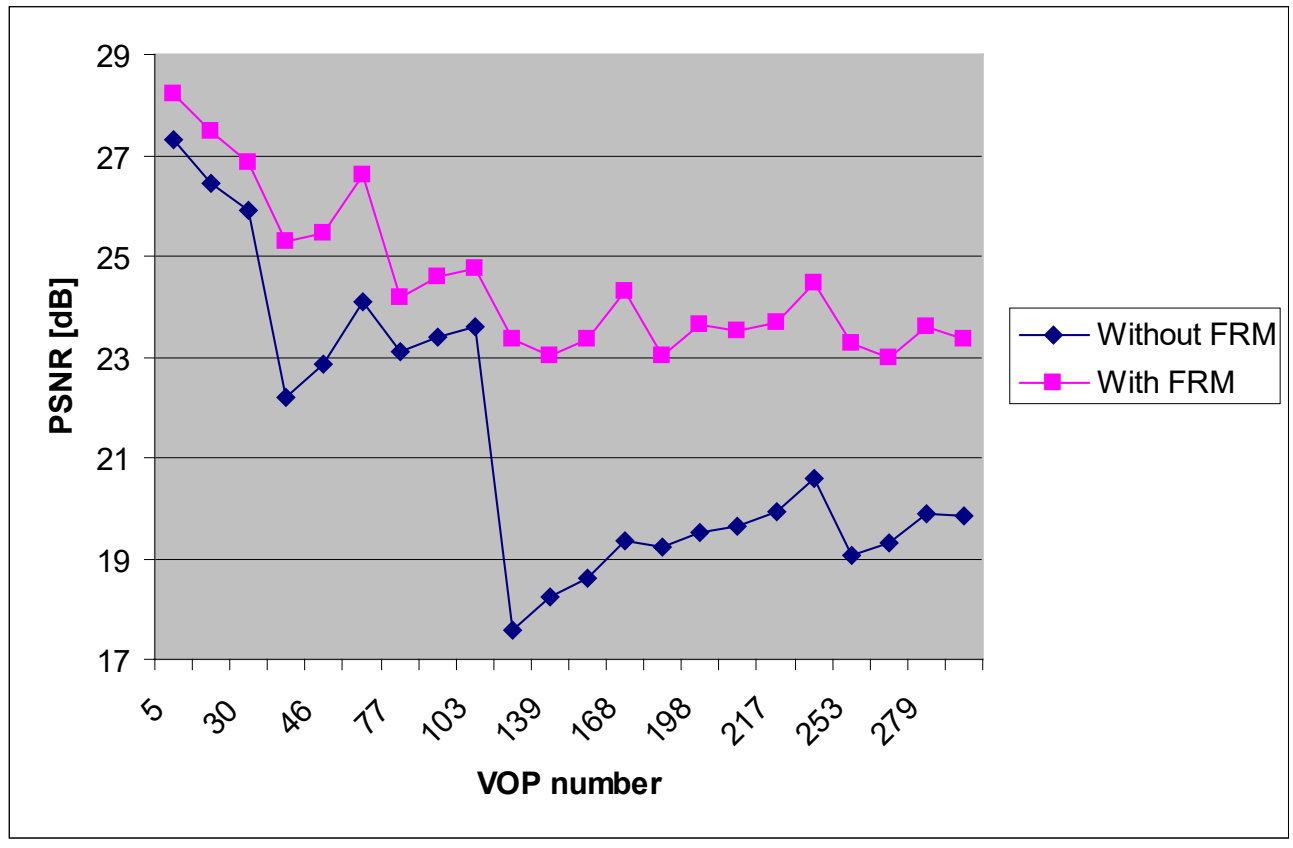

(c) COASTGUARD sequence. Frame Erasure Rate $=3 \%$, Residual Bit Error Rate $=$ $0.05 \%$.

Figure 10. Case A: PSNR of the enhancement layers under GPRS network conditions. 
As shown in Figure 10, the FRMs performed rather well under GPRS conditions. In the FOREMAN experiment, the enhancement in picture quality was up to $5 \mathrm{~dB}$, whereas in the other two experiments the quality gain was up to 2 and $6 \mathrm{~dB}$ respectively. This emphasises that the decoder was able to resynchronise its decoding process and recover from network error by utilising the FRMs.

Further, the subjective quality coincides with the presented objective quality. Figure 11 shows some decoded images corresponding to the experiments of Figure 10 above.

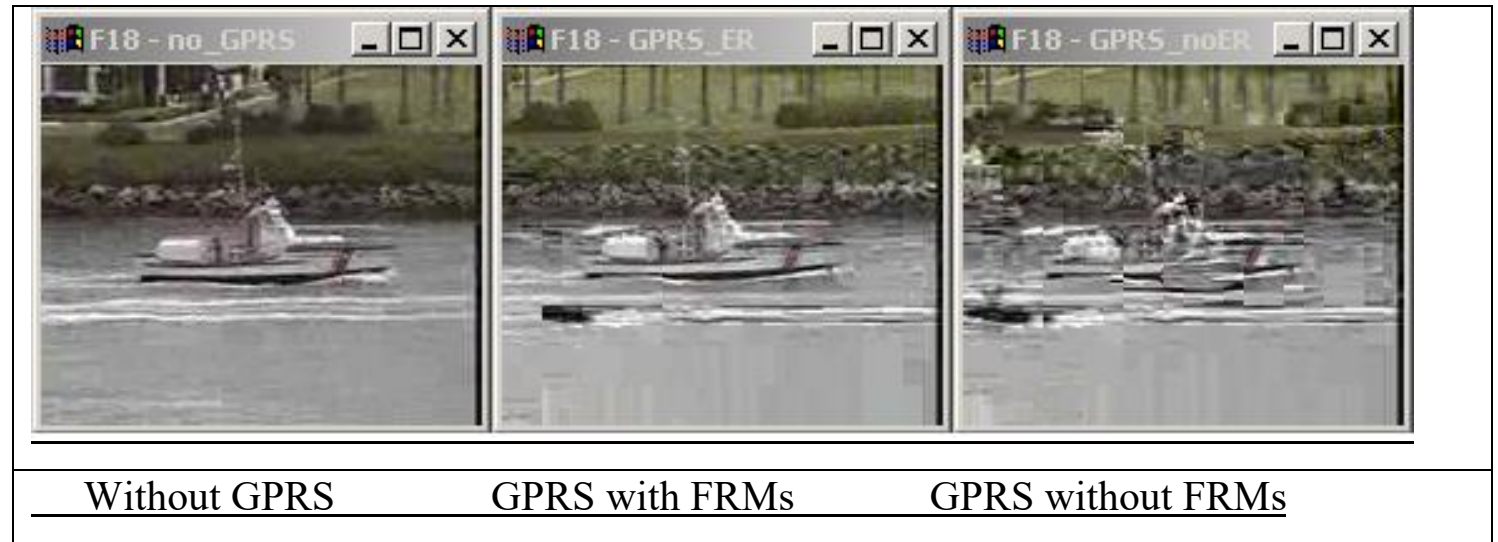

Figure 11. Subjective pictures corresponding to Figure 10 above.

Again it is evident from the figures that error recovery utilising the FRM is superior to the case where FRMs are not used.

\subsection{Case B results:}

In this case the enhancement VOPs are predicted from the base layer hence errors affecting the enhancement layer do not propagate in the temporal domain. Figure 12 plot the PSNR results for two test sequences but this time the prediction of the enhancement layers are restricted to the base layer. 


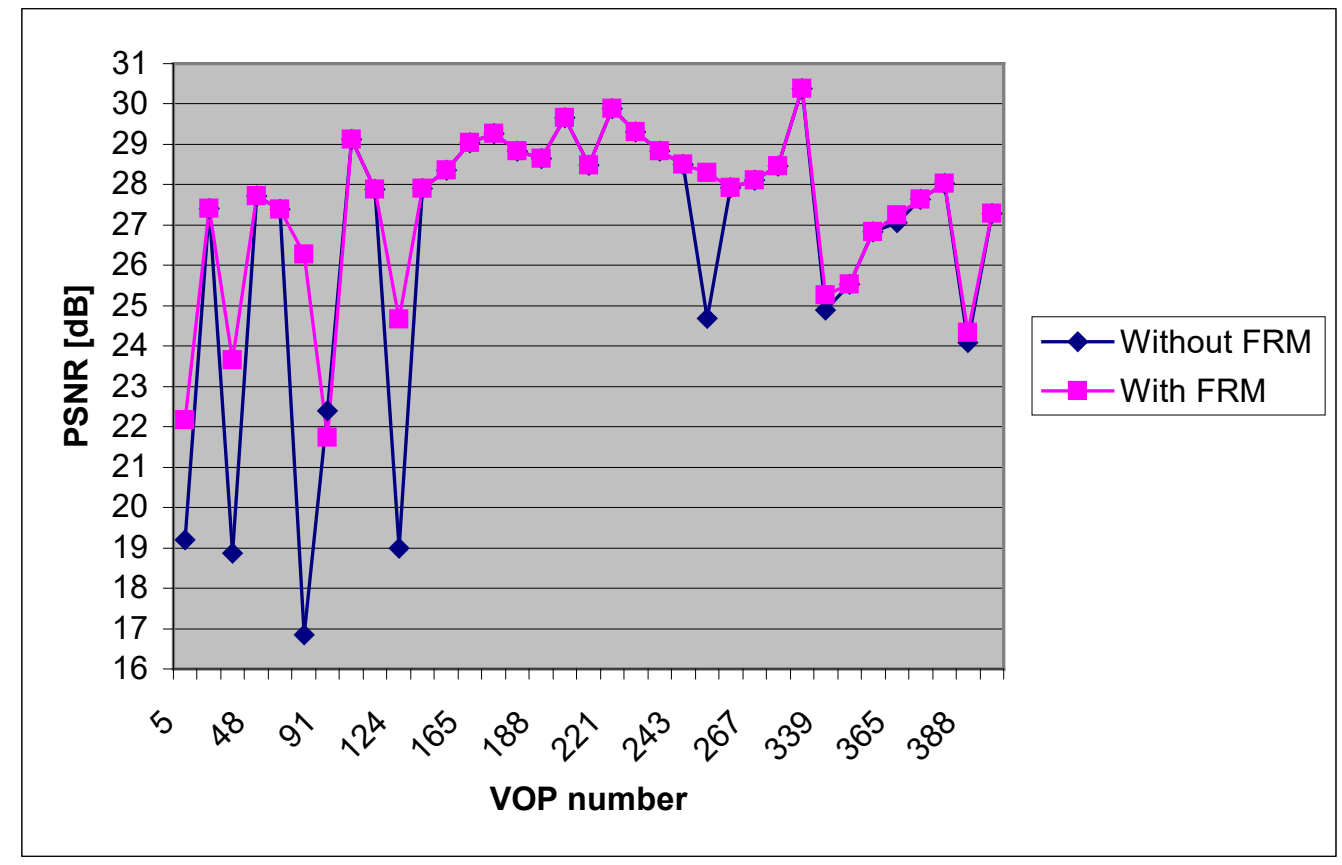

(a) FOREMAN sequence. Frame Erasure Rate $=3.4 \%$, Residual Bit Error Rate $=$ $0.055 \%$.

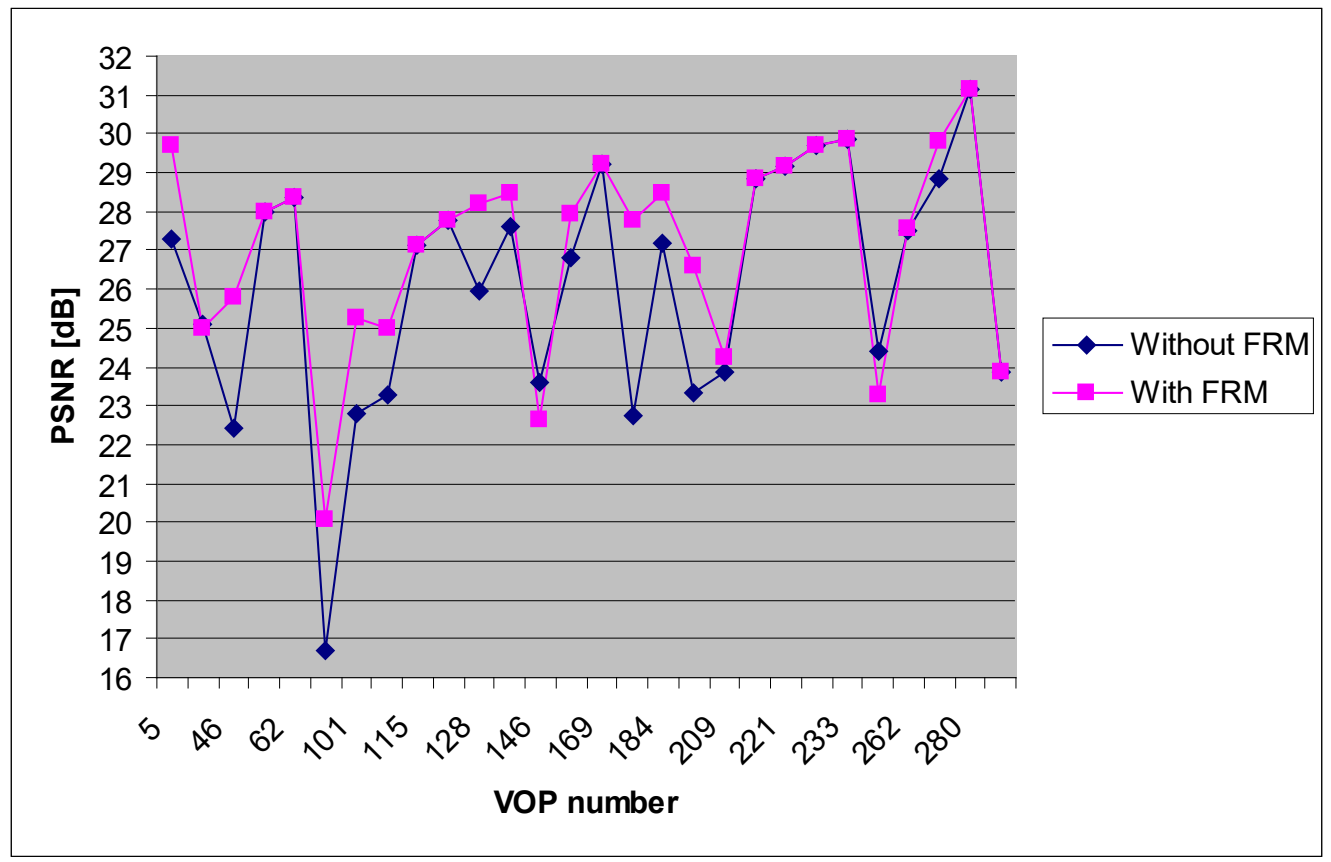

(b) COASTGUARD sequence. Frame Erasure Rate $=8.6 \%$, Residual Bit Error Rate $=$ $0.14 \%$.

Figure 12. Case B: PSNR of the enhancement layers under GPRS network conditions. 
In the figure, since the errors do not propagate in the temporal domain, the absence of the FRMs has a less dramatic effect. Nevertheless, it is still evident that the quality of recovered pictures utilising FRMs are still superior due to error localisations.

\section{Coding efficiency}

As mentioned earlier, interfering with the encoder's motion compensation decisions and bitrate control might result in lower coding efficiency. For our purpose since Constant Bitrate Coding (CBR) is used, the deficiency is mainly reflected in the percentage of coded VOPs. This section compares the coding results of the proposed FRM approach against the optimum or the free encoding approach. The comparison is given in Table 1 below.

\begin{tabular}{|c|c|c|c|c|c|c|c|}
\hline & \multicolumn{3}{|c|}{ Without FRM } & \multicolumn{3}{|c|}{ With FRM } & \\
\hline Sequence & $\begin{array}{l}\text { Bitrate } \\
\text { Kbps }\end{array}$ & No. VOPs & $\begin{array}{c}\text { PSNR } \\
\text { [dB] }\end{array}$ & $\begin{array}{l}\text { Bitrate } \\
\text { Kbps }\end{array}$ & $\begin{array}{c}\text { No. } \\
\text { VOPs }\end{array}$ & $\begin{array}{c}\text { PSNR } \\
{[\mathrm{dB}]}\end{array}$ & $\begin{array}{l}\text { Drop in } \\
\text { coded } \\
\text { VOPs }\end{array}$ \\
\hline Table & 19.93 & 35 & 30.9 & 19.61 & 31 & 31.17 & $-11.4 \%$ \\
\hline $\begin{array}{l}\text { Coastgu- } \\
\text { ard }\end{array}$ & 21.41 & 38 & 29.72 & 21.49 & 36 & 29.02 & $-5.2 \%$ \\
\hline Weather & 20.96 & 33 & 27.31 & 21.08 & 34 & 28.41 & $+3 \%$ \\
\hline Foreman & 21.1 & 46 & 28.82 & 21.38 & 38 & 27.89 & $-17.4 \%$ \\
\hline
\end{tabular}

Table 1. Effect of FRM on coding efficiency.

As shown in the Table, the most significant deviation is reflected in the number of coded VOPs. The table shows one exception though which was generated using the WEATHER test sequence. Recall from section 3.1.1 that the lower blocks of the MB row preceding a FRM and the upper blocks of the FRM row itself are forced to be coded without motion compensation, that is using a nil motion vector. Therefore, depending on the temporal activity, the suitability of FRMs varies from one sequence to another.

Recall that in FRMs, the upper $8 \times 8$ row of blocks of a VP and the immediate preceding row of $8 \times 8$ blocks are forced to no motion compensation. Thus in a low temporal activity sequence, a high percentage of skipped MBs is to the advantage of employing FRMs. 


\section{Limitations of the syntax friendly approach:}

The limitations of the proposed syntax friendly approach to error resiliency are summarized here:

1. The drawback of FRMs is that the MPEG-4 visual specification restricts the use of stuffing bits to P-VOPs. Hence the coding gain achieved through the bidirectional prediction cannot be utilized.

2. The interference with the last coded DCT coefficient of a MB preceding a VP to ensure byte-alignment of stuffing bits degraded the image quality as well.

3. The interference with the quantization step sizes to ensure the correct resetting of quant_value at the beginning of each VP affected the accuracy bitrate control algorithm.

4. The interference with the macroblock coding modes to ensure correct resetting of MV predictors at the beginning of each VP resulted in coding deficiencies. The same argument applies for resetting the AC and DC predictors.

5. The alternative solution to Header Extensions manipulates an informative part of the ISO MPEG-4 standard and is considered unfair to compliant decoders.

Based upon such limitations it is evident that syntax changes to incorporate the error resiliency tools in the enhancement layers of the SSP profile are needed. The syntax changes which have been approved by the ISO MPEG-4 committee are elaborated upon next.

\section{Syntax modifications for SSP known as Error Resilient SSP (ER-SSP).} This section highlights the changes proposed to and approved by the ISO MPEG-4 committee to incorporate the error resiliency tools in the enhancement layer of the SSP profile.

\subsection{Modifications to the Header Extension Tool}

The HE was originally designed for use in the Video Packet Header of the base layer hence, only the header information of the base is replicated. If the HE is to be employed in the enhancement layer then vital header information of the 
enhancement layer has to be replicated as well. Keeping in mind that the purpose of the HE is to facilitate independent decoding of Video Packets, it follows that if Video Packets were incorporated in enhancement layers of the Simple Scalable Profile then each Video Packet must be able to identify its reference VOPs or source of prediction. In other words the 'ref_select_code' must be added to the HE.

Note that the encoder selects the 'ref_select_code' on a VOP basis, which implies that this field can be changed from one VOP to another according to the underlying implementation.

In the spatial scalability however, the 'ref_select_code' is predetermined. It is fixed to ' 11 ' and ' 00 ' for $\mathrm{P}$ and $\mathrm{B}$ VOPs respectively. Hence replicating it in the $\mathrm{HE}$ might not be as important as with the temporal scalability case. Nevertheless, since the 'ref_select_code' is predetermined for spatial scalability, replicating it in the HE can serve as an error detection feature just as the case with the 'marker_bit'.

\subsection{Modification to the existing bitstream syntax}

The use of the three error resilience tools in the enhancement layer requires amending its decoding process to make it similar to that of the base layer. That is, instead of calling combined_motion_shape_texture(), the enhancement layer VOPs are decoded by looping through the motion_shape_texture() and video_packet_header() functions as defined in section 6.2 "Visual bitstream syntax" in the MPEG-4 visual standard.

More specifically, section 6.2.5 "Video Object Plane and Video Plane with Short Header, VideoObjectPlane()" of the MPEG-4 visual standard the syntax was from: 


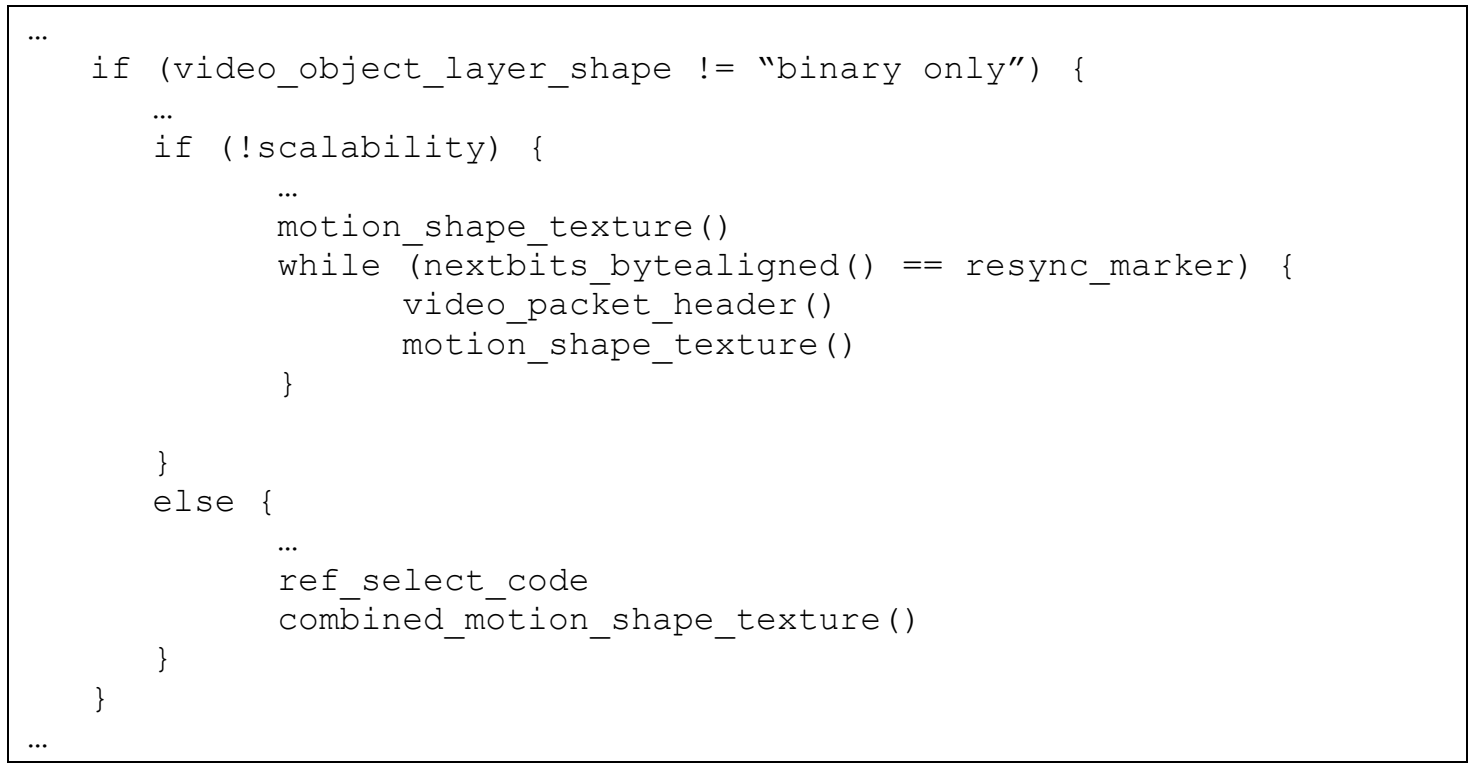

To:

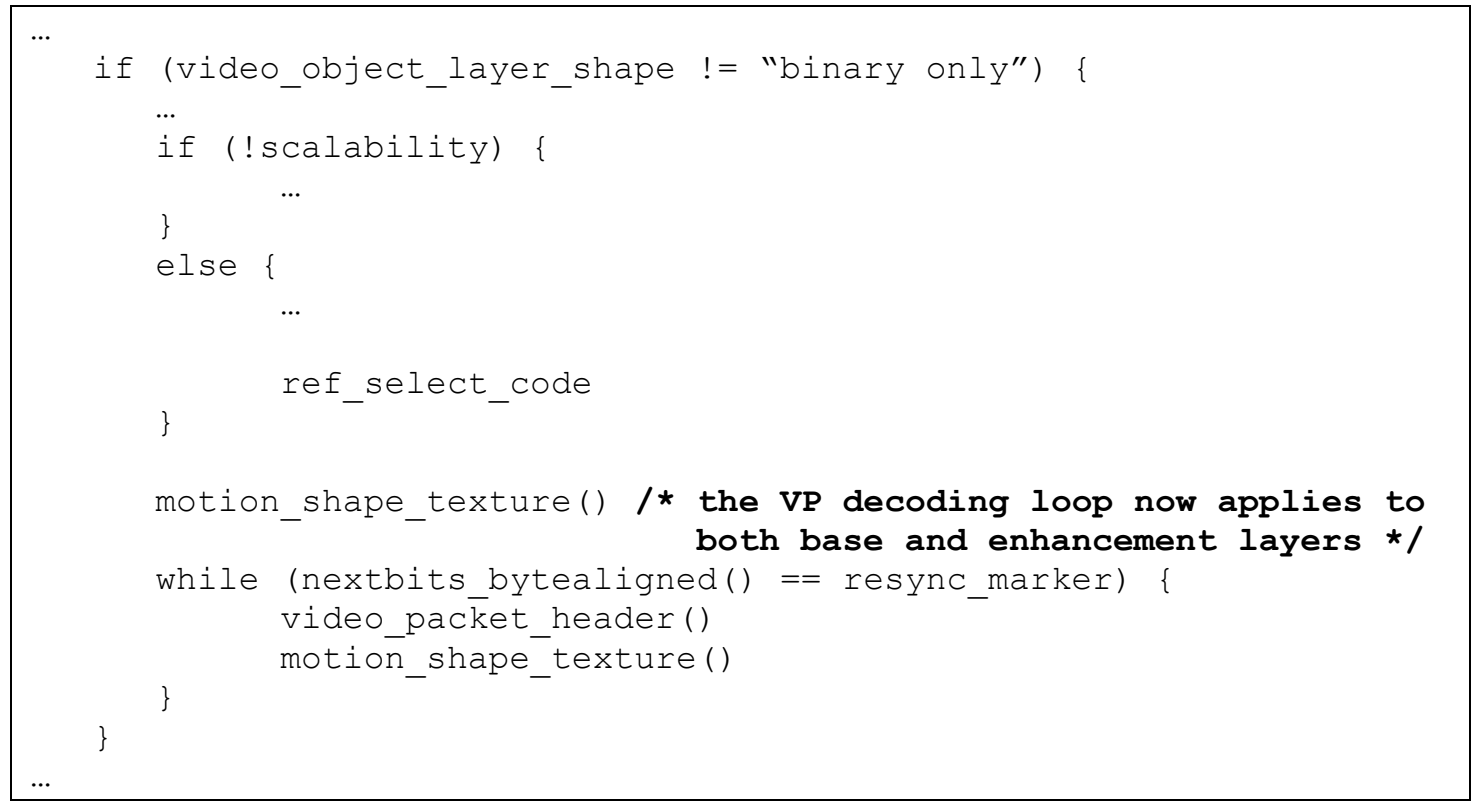

Additionally for amending the HE of the Video Packet Headers, section 6.2.5.2 "Video Object with Short Header, video_packet_header()" of the MPEG-4 visual standard the syntax was changed to: 


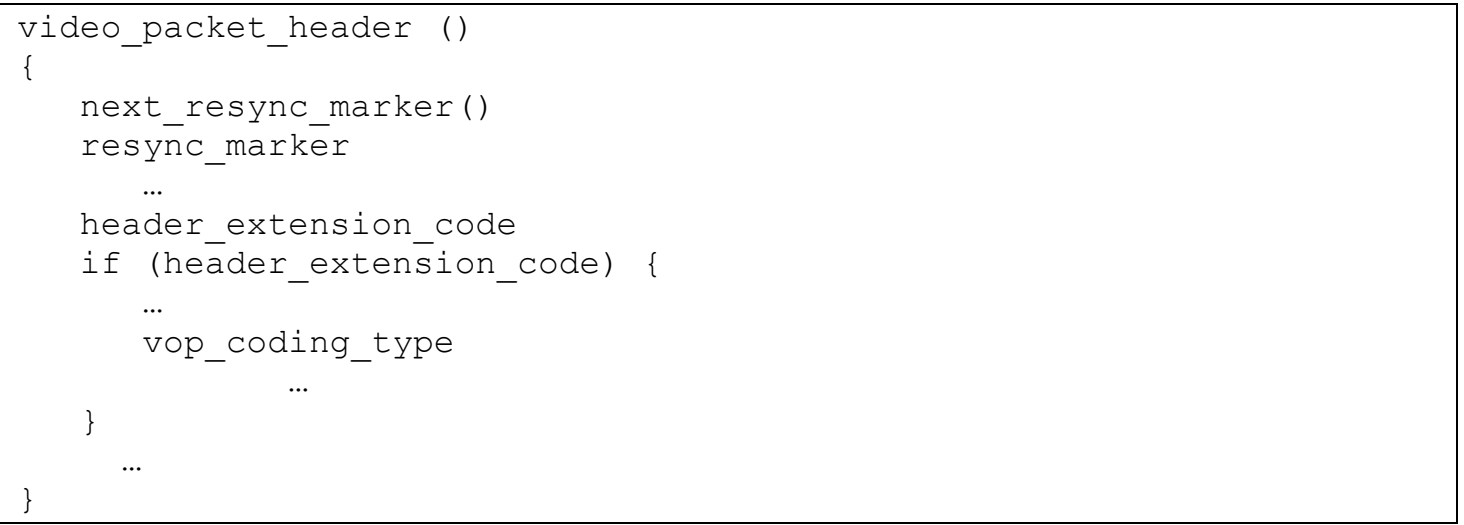

To:

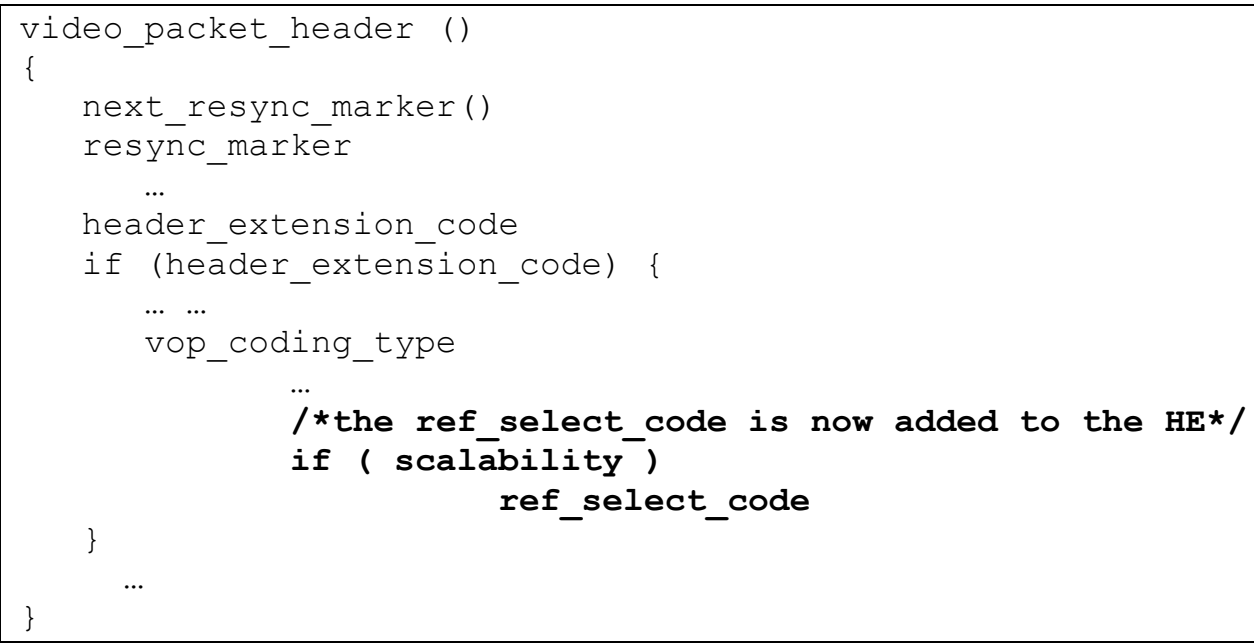

Note that neither of these changes will affect decoding of ordinary SSP video. In any bitstream without error resilience, the first call to motion_shape_texture() will decode a complete VOP, so the call to video_packet_header() will not be made. Lastly, recall that in SNR and spatial scalability modes, P-VOPs of the enhancement layer can be predicted from the base provided that no motion compensation is used thus no motion vectors are transmitted. This is reflected in the 'ref_Select_code' value of ' 11 '. If the data partitioning tool is employed then no motion vectors are available for decoding before the 'motion_marker' in the video packet. Hence this should be reflected in the new syntax as shown below. The 'data_partitioned_p_vop ()' is modified from: 


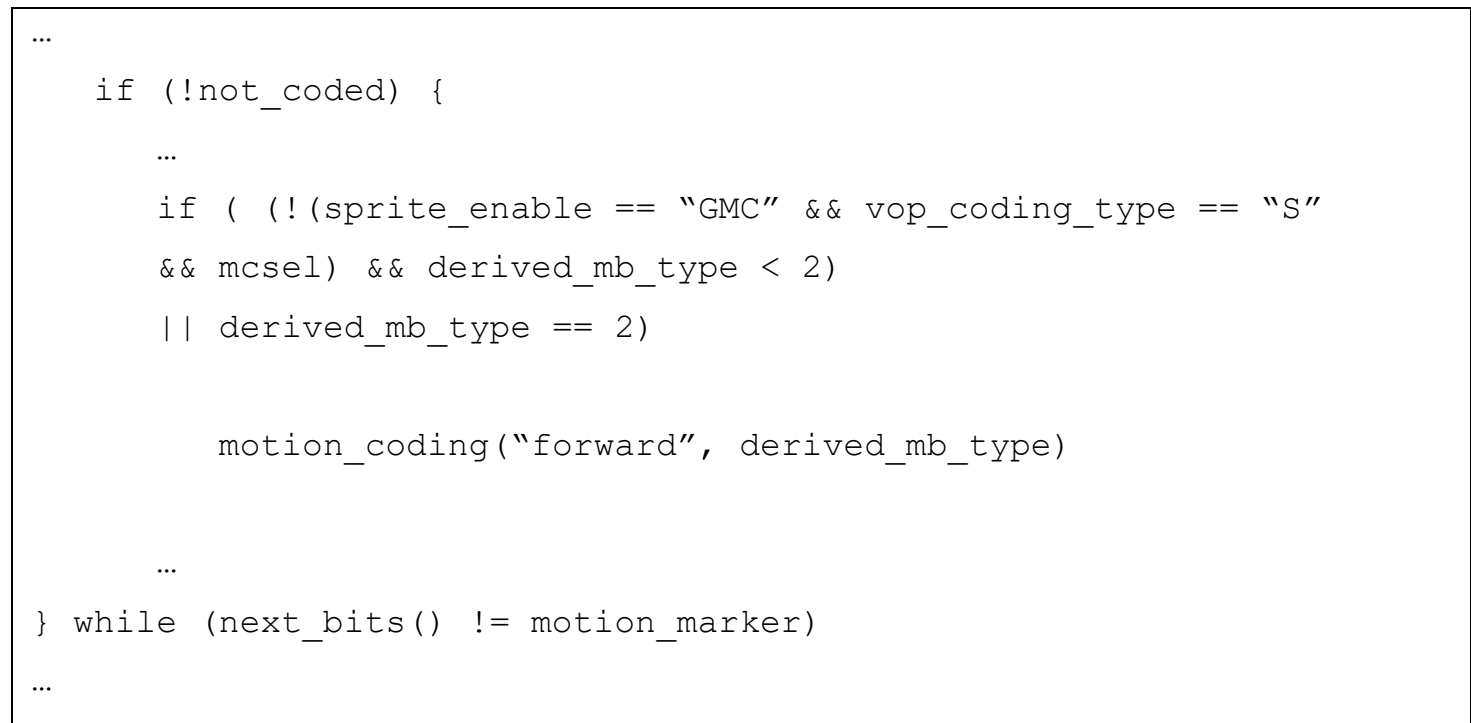

To:

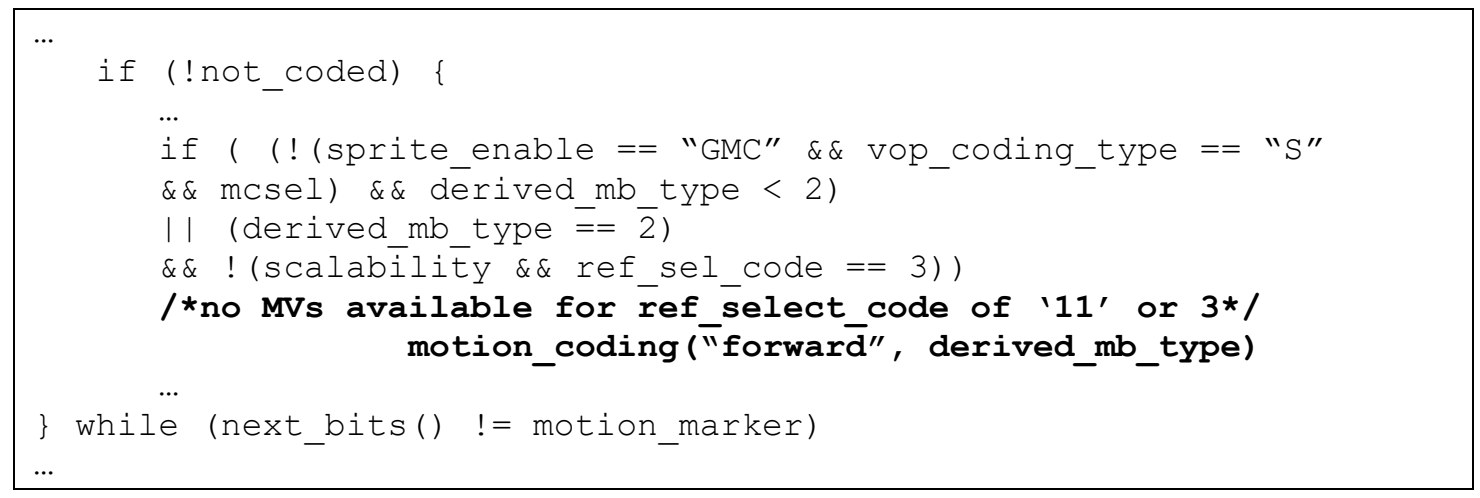

\subsection{Overhead of applying the ER tools to the enhancement layers}

For a QCIF sequence, the use of Video Packet Headers in enhancement BVOPs requires around 55 bits. However, since Video Packets reset the predictors, including $A C / D C$, motion vectors and so on, the extra overhead is generally sequence dependent and can only be estimated by means of empirical testing.

In this experiment Video Packet Headers are injected at a periodic rate of 800 bits (here the HEC exists once per VOP) as the case with the sequences presented in the above experiments. A mixture of 52 short test sequences comprising 3329 frames is used. The resultant extra overhead is given in Table 2. 


\begin{tabular}{|c|c|c|c|}
\hline & $\begin{array}{c}\text { Video Packet } \\
\text { Headers }\end{array}$ & Data partitioning & RVLC \\
\hline $\begin{array}{c}\text { B-VOPs } \\
\text { (enhancement - ER } \\
\text { SSP profile). }\end{array}$ & $4.2 \%$ & N/A & N/A \\
\hline $\begin{array}{c}\text { P-VOPs } \\
\text { (enhancement - ER } \\
\text { SSP profile) }\end{array}$ & $3.9 \%$ & $6 \%$ & $6.18 \%$ \\
\hline $\begin{array}{c}\text { P-VOPs (base-SP } \\
\text { profile) }\end{array}$ & $3.77 \%$ & $5.8 \%$ & $6.2 \%$ \\
\hline
\end{tabular}

Table 2. Excessive overhead cased by the error resilience tools

As the table shows incorporating the error resilience tools in the enhancement layers incurs in an overhead similar to that of the base layer. This emphasizes the fact that these tools are equally applicable to both layers.

\subsection{MPEG-4 Syntax Amendments Verification Testing}

The final stage of the MPEG amendment submission process is verification testing. The goal is to provide evidence that the amendment is necessary, and to advertise the availability and utility of the amendment within the MPEG community.

The subjective test method used in this verification effort was the new MMDSCQE (Multi Media Double Stimulus Continuous Quality Evaluation) derived from the DSCQE method previously used to verify the first error resilience tools in MPEG [14] and [15]. The new method differs from the previous one mainly in the usage of a progressively scanned display (i.e. a PC monitor LCD or CRT); also in this case the error free video and the error prone video are displayed in two side by side windows as shown in Figure 13. 


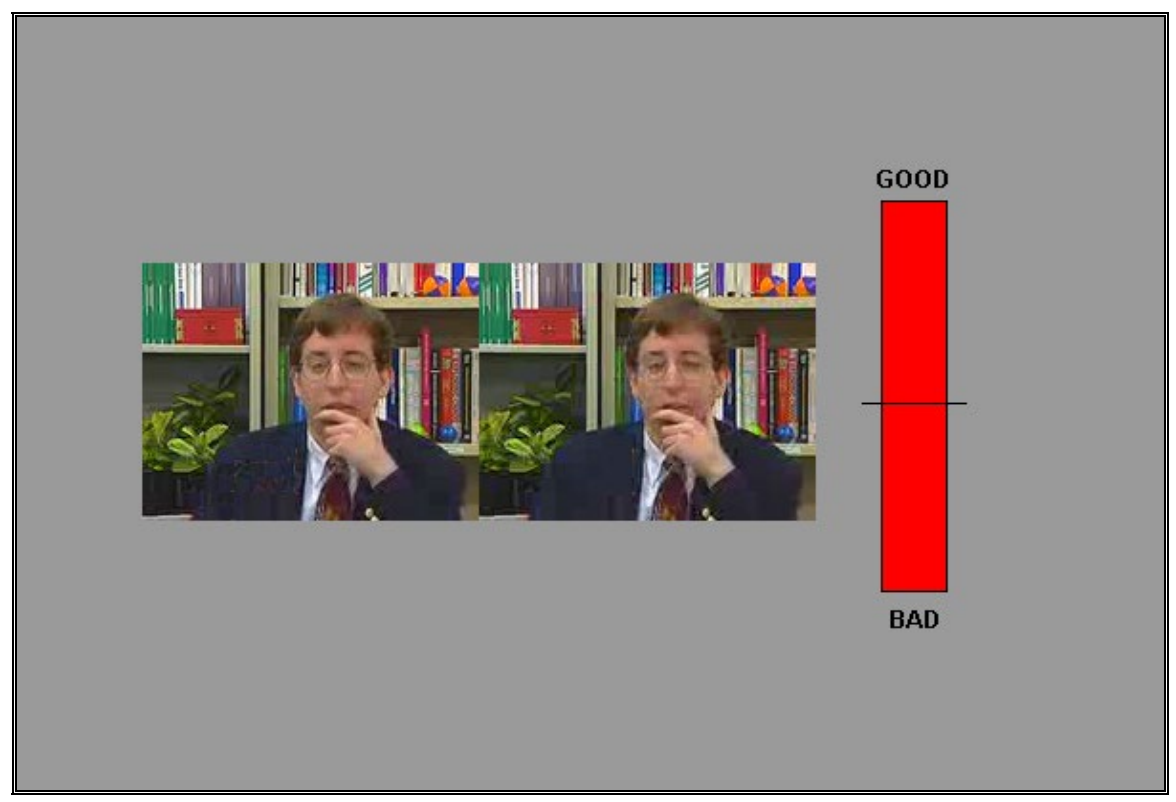

Figure 13. Deployment of the Multi Media Double Stimulus Continuous Quality Evaluation method.

The subject uses the PC mouse to provide his/her quality observation during the test. A bar placed, on the right side of the two windows containing the images, provides the subjects with feedback on the currently observed quality. Moving the mouse up and down, the bar moves accordingly following the actions of the subject.

The error patterns used to corrupt the bitstreams were obtained from a GPRS link layer simulator. For these tests, the carrier to interference ratio was set to 12 . This provides a reasonable representation of field conditions, without completely overwhelming the non-error resilient codec. The latter point is important in that, for a valid user trial, we do not want either quality measure to bottom out as soon as errors are added to the bitstream.

In order to isolate the enhancement layer error resilience for the user trials, the base layer of each video was transmitted error-free. The subjective evaluation results are shown in Figure 14. 


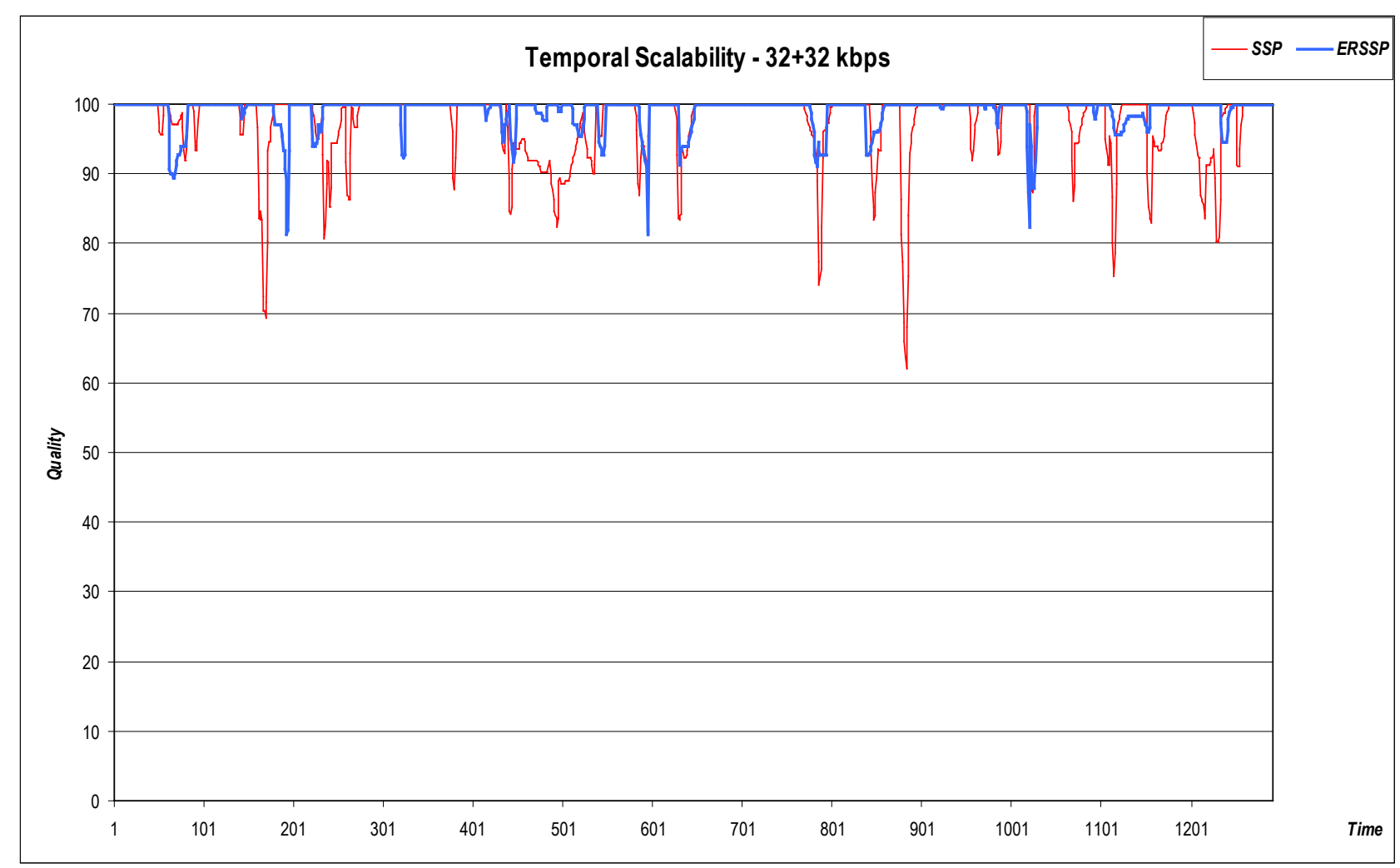

(a) Temporal scalability testing $32 \mathrm{kbit} / \mathrm{s}$ per layer

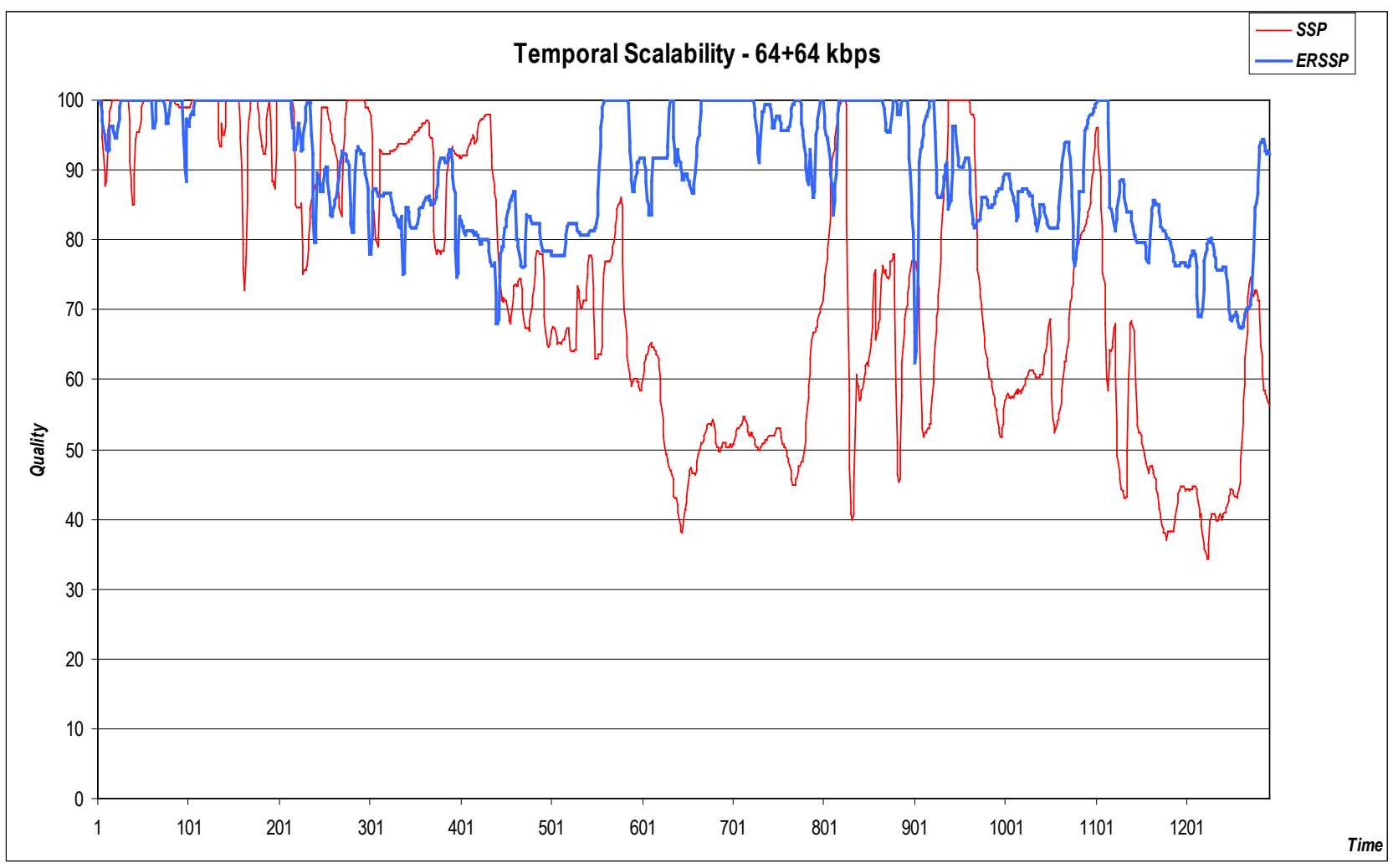

(b) Temporal scalability testing $64 \mathrm{kbit} / \mathrm{s}$ per layer 


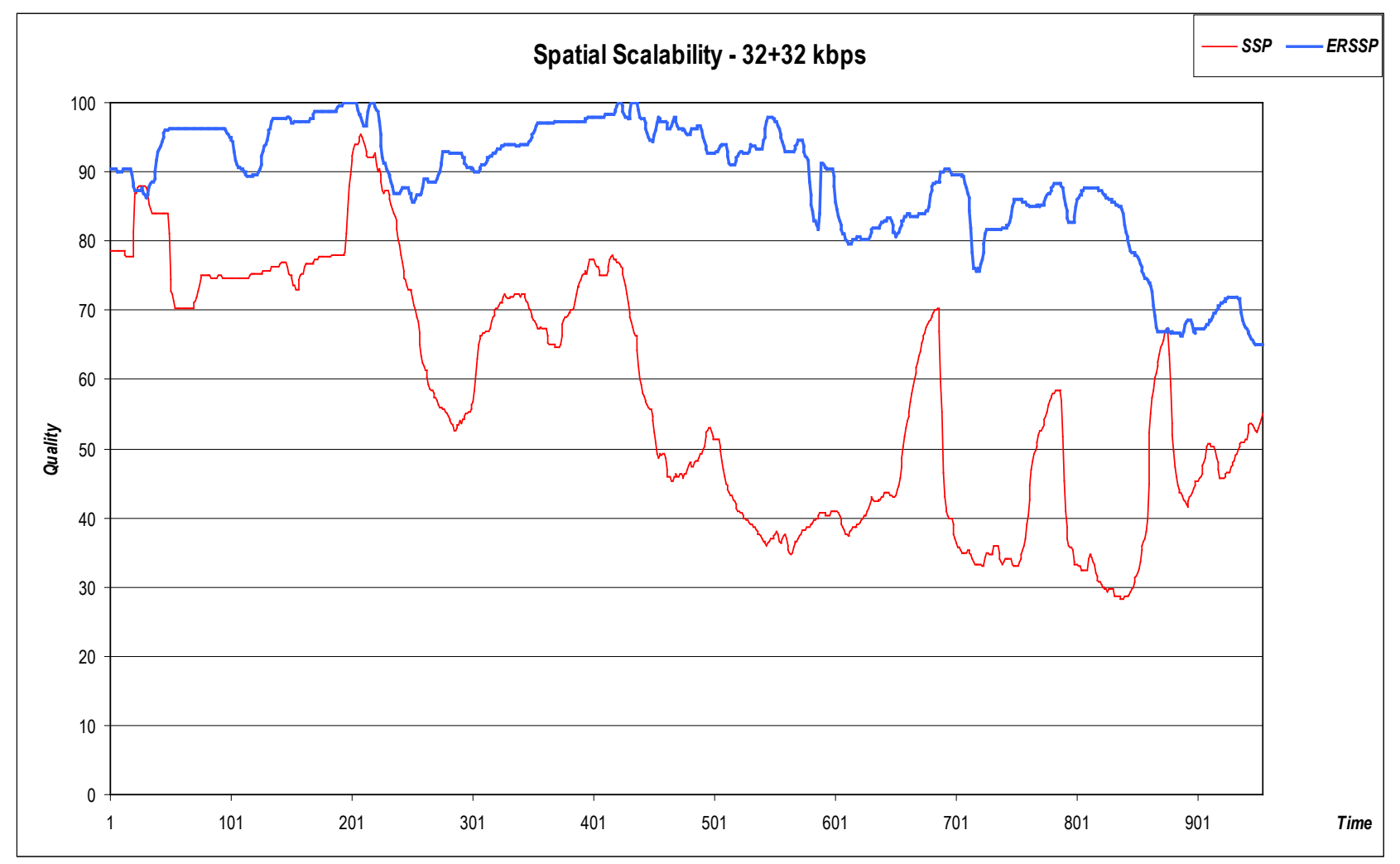

(c) Spatial scalability testing $32 \mathrm{kbit} / \mathrm{s}$ per layer

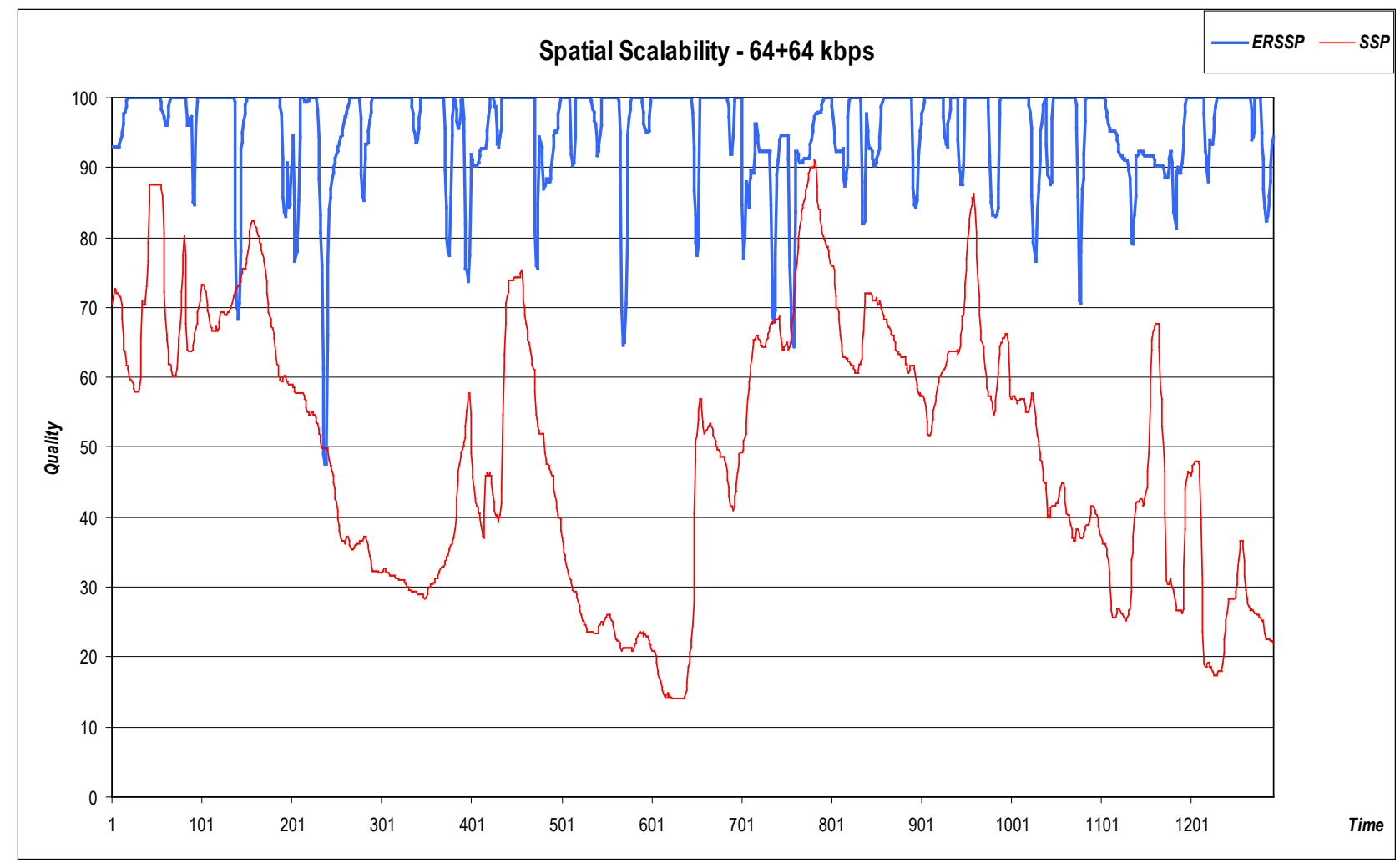

(d) Spatial scalability testing 64kbit/s per layer 
Figure 14. Results of the subjective evaluation of the ER-SSP profile.

Test QCIF sequences are used; MaD900 and Deadline, each of them running for between 45 seconds and 1 minute. The original test material was at 30fps. For the spatial scalability case it was downsampled to $10 \mathrm{fps}$.

In the case of temporal scalability the base layer was sampled at $5 \mathrm{fps}$. For the $64 \mathrm{kbps}$ case, the enhancement layer added 2 frames per base layer frame, to increase the rate to $15 \mathrm{fps}$. For the $32 \mathrm{kbps}$, the enhancement layer added 1 frame per base layer frame, to take the rate to $10 \mathrm{fps}$. In both cases the rate controller was again allowed to drop frames to meet the bit-rate targets.

Figure 14 demonstrates the superiority of the ER-SSP method over unprotected scalable layers under all tested conditions. The amendment was therefore accepted for inclusion in the MPEG-4 Visual standard.

\section{Conclusions:}

The lack of error resiliency tools in the enhancement layers of the SSP profile prevented its deployment in mobile communications. The paper proposed two solutions to alleviate this problem. A syntax friendly approach was proposed in which video packets are emulated whilst adhering to the syntax of the underlying profile. Stuffing bits are used to emulate synchronization markers. Various techniques are then proposed to reset all the predictors at the boundaries of video packets such as motion vectors, quantizers and AC/DC predictors. This approach was shown to add transmission error robustness to the enhancement layers nevertheless it was limited to P-VOPs and added numerous coding constrains.

Based upon the limitations of the syntax friendly approach, a syntax amendment solution was proposed. It was shown that the error resiliency tools of the base layer are applicable to the enfacement layer with few modifications. For

instance, the 'ref_select_code' which indicated the source of VOP prediction was added to the HE tool. Additionally the syntax of the data partitioning tool was 
slightly modified to accommodate P-VOPs predicted from the base layer without motion compensation. The excessive overhead of amending the syntax was shown to be similar to that of the base layer. Formal subjective testing was then employed to verify the amendments. Subsequently, the modifications were accepted by the ISO/IEC MPEG-4 committee and resulted in a new profile known as the Error Resilient Simple Scalable Profile (ER-SSP).

\section{References:}

[1] ISO/IEC JTC 1/SC 29/WG 11 "Information technology - Generic coding of audio-visual objects - Part2: Visual," N4350, Sydney, July 2001

[2] T. Shanableh and M. Ghanbari "Loss Concealment using B-pictures Motion Information," IEEE Transactions on Multimedia, 5(2), pp. 257-266, June 2003 [3] T. Shanableh and M. Ghanbari, "Backward tracking of B-pictures bidirectional motion for interframe concealment of anchor pictures," Proc. of ICIP, v 3, 2000, $p$ 396-399, Vancouver, September 2000

[4] P. Hobson and T. Shanableh "Request for use of error resilience tools with MPEG-4 simple scalable profile," ISO/IEC JTC1/SC29/NG11 m7651, Pattaya, November 2001

[5] T. Shanableh and P. Hobson "A proposal for syntax amendments to use the error resilience tools in the MPEG-4 Simple Scalable Profile," ISO/IEC JTC1/SC29/WG11 m7919, Jeju, March 2002.

[6] ISO/IEC 14496-2:2004/Amd.1, "AMENDMENT 1: Error resilient simple scalable profile," June 2004.

[7] R. Yan, F. Wu, S. Li and R. Tao "Error resilience methods for FGS video enhancement bitstream," First Pacific Rim conference on multimedia, IEEE PCM2000 Dec $13-152000$

[8] R. Yan, F. Wu, S. Li, R. Tao, Y. Wang and Y.-Q. Zhang "Error robust coding for the FGS enhancement bitstream," Third International Conference on Information, Communications, Singapore, October, 2001

[9]J. Brailean, "MPEG-4 Error Resilience Tools, "ITU - SG-16, Document Q11C-38, Eibsee, December 1997 
[10] R. Talluri, "Error-Resilient Video Coding in the ISO MPEG-4 Standards," IEEE Communications Magazine, 26(6), pp. 112-119, June 1998

[11] J. Bongsoo, H. Y. Hooi, J. Byeungwoo, K. M. Don and C. Song-In, "Error resilient performance evaluation of MPEG-4 and H.264,"Proc. of SPIE - The International Society for Optical Engineering, v 5150 II, 2003, p 1050-1061 [12] J. Wen, and J. Villasenor, "Reversible Variable Length Codes for Efficient and Robust Image and Video Coding", Proc. of 1998 IEEE Data Compression Conference, p.471, Snow Bird, UT, USA, 1998.

[13] W. Featherstone and D. Molkdar "System level performance evaluation of GPRS for various traffic models," Vehicular Technology Conference, IEEE-VTS Fall VTC 2000. 52nd, vol. 6, pp 2648 -2652, Boston, USA, September 2000 [14] ISO/IEC JTC 1/SC 29/WG 11 w2165, "Specification of formal verification tests on video error resilience," $43^{\text {rd }}$ MPEG meeting, Tokyo, March 1998 [15] ISO/IEC JTC 1/SC 29/WG 11 w1829, "Plan for March 1998 Error Resilience Verification Test," 40 ${ }^{\text {th }}$ MPEG meeting, Stockholm, July 1997 
Appendix A: a possible set of VLC codes to ensure byte-alignment of FRMs.

\begin{tabular}{|l|c|c|c|c|}
\hline $\begin{array}{c}\text { Bits to } \\
\text { complete } \\
\text { byte }\end{array}$ & VLC & Last & Run & Level \\
\hline 7 & $001110 \mathrm{~s}$ & 1 & 2 & 1 \\
\hline 6 & $110 \mathrm{~s}$ & 0 & 1 & 1 \\
\hline & $000011001 \mathrm{~s}$ & 1 & 0 & 2 \\
\hline 5 & $10 \mathrm{~s}$ & 0 & 0 & 1 \\
\hline & $000011001 \mathrm{~s}$ & 1 & 0 & 2 \\
\hline 4 & $00000000101 \mathrm{~s}$ & 1 & 0 & 3 \\
\hline 3 & $01101 \mathrm{~s}$ & 0 & 3 & 1 \\
\hline & $0111 \mathrm{~s}$ & 1 & 0 & 1 \\
\hline 2 & $000011001 \mathrm{~s}$ & 1 & 0 & 2 \\
\hline 1 & $110 \mathrm{~s}$ & 0 & 1 & 1 \\
\hline & $0111 \mathrm{~s}$ & 1 & 0 & 1 \\
\hline
\end{tabular}

Table A-1. VLC codes to add for DCT blocks preceding a FRM.

\section{Examples:}

E1. If the number of bits needed to complete a byte is 7 then the VLC '0011 10s' is added to the DCT block. Since this VLC has the LAST flag set then no further action is required.

E2. If the number of bits needed to complete a byte is 6 then the VLC '110s' can be appended, in this case 2 or $2+8$ more bits are needed. Hence the VLC ' 0000 $11001 \mathrm{~s}^{\prime}$ can be added with the LAST flag set. In this case the total added bits are byte aligned. 


\section{Appendix B: an algorithm to reinitialize the quantization index after a FRM.}

As explained in section 3.2.3 above, the aim is to reinitialize the quantization index after a FRM to an absolute value which is the 'vop_quant' without violating the existing bitstream syntax.

In MPEG-4 P-VOPs, unlike MPEG-2, the variation in quantization from one MB to another is restricted to the range of $[-2,2]$. Hence the quant of a MB following a FRM cannot be set to the value of 'vop_quant' unless the difference between the latter and the quant of the previous MB falls within the specified range.

In other words, the aim is to ensure that the quant of the MB preceding a FRM, denoted by $Q_{i-1}$ (a distance of one macroblock from the FRM), is at most 2 steps apart from 'vop_quant'. For this to hold, $Q_{i-2}$ (a distance of two macroblock from the FRM), should be at most be 4 steps apart from 'vop_quant' and so forth until we reach back to $Q_{i-16}$ which should be at most be 32 steps apart from 'vop_quant'. Since the valid quant range is $[1,31]$ then no further MBs need to be inspected.

Hence, for a QCIF picture, if the FRMs are injected every two rows of macroblocks then each MB will have the following distances from the FRMs shown in Figure B-1. Note that there is no need to exceed 16 locations, as $16^{*} 2$ is larger than the valid quant range of [1,31].

\begin{tabular}{|c|c|c|c|c|c|c|c|c|c|c|}
\hline 1 & 2 & 3 & 4 & 5 & 6 & 7 & 8 & 9 & 10 & 11 \\
\hline 16 & 16 & 16 & 16 & 16 & 16 & 16 & 15 & 14 & 13 & 12 \\
\hline 11 & 10 & 9 & 8 & 7 & 6 & 5 & 4 & 3 & 2 & 1 \\
\hline 0 & 16 & 16 & 16 & 16 & 16 & 16 & 15 & 14 & 13 & 12 \\
\hline 11 & 10 & 9 & 8 & 7 & 6 & 5 & 4 & 3 & 2 & 1 \\
\hline 0 & 16 & 16 & 16 & 16 & 16 & 16 & 15 & 14 & 13 & 12 \\
\hline 11 & 10 & 9 & 8 & 7 & 6 & 5 & 4 & 3 & 2 & 1 \\
\hline 0 & 16 & 16 & 16 & 16 & 16 & 16 & 16 & 16 & 16 & 16 \\
\hline 16 & 16 & 16 & 16 & 16 & 16 & 16 & 16 & 16 & 16 & 16 \\
\hline 16 & 16 & 16 & 16 & 16 & 16 & 16 & 16 & 16 & 16 & 16 \\
\hline
\end{tabular}

Figure B-1. MB distance map with respect to Faked Resynchronization Markers (FRM). Location ' 0 ' indicates the location of the FRM. 
Now at each MB location the following inequality is examined:

$$
\left|Q_{i, j}-v o p_{-} q u a n t\right| \leq \text { dist }_{i, j} \times 2
$$

Where $Q_{i j}$ is the quant of the MB of the $i^{\text {th }}$ row and $j^{\text {th }}$ column. Likewise, dist ${ }_{i j}$ is derived from Figure B-1 according to the $i^{\text {th }}$ and $j^{\text {th }}$ coordinates.

If the inequality does not hold then the underlying quant is modified according to the following formula:

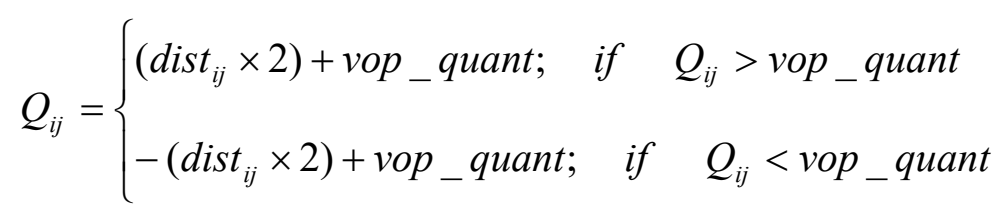

Obviously if $Q_{i j}=v o p_{-} q u a n t$ then no action is needed.

As such, the quant of the MB located just before a FRM will always be at most two steps away from 'vop_quant' and therefore the quant of MB after a FRM can now be initialized to the value of 'vop_quant'. 